\title{
Investigation on the Effects of Structural Dynamics on Rolling Bearing Fault Diagnosis by Means of Multibody Simulation
}

\author{
Reza Golafshan (D), Georg Jacobs, Matthias Wegerhoff, Pascal Drichel, and Joerg Berroth \\ Faculty of Mechanical Engineering, Institute for Machine Elements and Systems Engineering (MSE), RWTH Aachen University, \\ Schinkelstr. 10, 52062 Aachen, Germany
}

Correspondence should be addressed to Reza Golafshan; reza.golafshan@imse.rwth-aachen.de

Received 23 October 2017; Accepted 22 January 2018; Published 13 March 2018

Academic Editor: Paolo Pennacchi

Copyright (C) 2018 Reza Golafshan et al. This is an open access article distributed under the Creative Commons Attribution License, which permits unrestricted use, distribution, and reproduction in any medium, provided the original work is properly cited.

\begin{abstract}
The present study aims to combine the fields modal analysis and signal processing and to show the use of Frequency Response Function (FRF), as a vibration transfer path, in enhancing reliability and abilities of the next generation vibration-based rolling bearing condition monitoring (CM) systems in complex mechanical systems. In line with this purpose, the hereby-presented paper employs an appropriate numerical model, that is, Multibody Simulation (MBS) of a vehicle's drivetrain as a manner for numerical modal and structural analyses. For this, first, the principles of vibration-based bearing fault detection are reviewed and presented. Following that, a summary of MBS modelling and validating strategies are given. Then, the validated MBS model is used as a case study for further investigations. The results can confirm existence of challenges in fault detection of rolling bearings, in particular in complex mechanical systems. In further discussions, the capability of FRFs in fault localization and determination of ideal sensor positions is discussed in some detail. Finally, concluding remarks and suggestions for future works are summarized.
\end{abstract}

\section{Introduction}

In a vibration-based fault detection and fault diagnosis system, mechanical damage is referred to changes on geometric properties of the analyzed machine. These sudden changes may generate additional dynamical forces acting in the system. Then, based on the system responses, damage/faults can be detected and localized. Since all machines vibrates even in their good operational condition due to interactions between machine components, the captured vibrations signals are required to analyze using signal postprocessing techniques. Many diagnostic procedures assume a linear behavior, and thus each individual spectral component (or a group of them) is supposed to relate to a specific dynamic force in the corresponding machine [1]. Therefore, captured time domain vibration signals need to be transformed to the frequency domain to distinguish the various sources and to detect the probable faults, accordingly [2]. However, due to the mechanical complexities and varying operating conditions, it is not always possible to capture, separate, process, and identify the faulty signals, and this could be the case in many complex systems; hence, an overview of dynamics of the analyzed structure/machine may be needed for the reliable and next generation vibration-based fault detection systems $[3,4]$. It is known that the captured vibration signals contain structural properties (i.e., vibration transfer path) of the system being analyzed and they are assumed to remain same under varying operating conditions (e.g., torque loads and rotating speeds). Thus, a prestudy on dynamics of the analyzed structure/machine may be necessary to develop suitable and reliable condition monitoring (CM) and detection techniques to detect and diagnose various failures and achieve cost savings to the industry. The modal behavior of a system can be a useful insight to gain beneficial information on the system response to various possible faults and failures. According to the findings in the literature, modal domain features (i.e., natural frequencies, mode shapes, and damping ratios) are widely used in the field Structural Health Monitoring (SHM), in particular for composite and large civil structures [5]. By tracking and analyzing changes over time the presence of a failure, from crack propagation to unbalance localization, can be approved. However, for mechanical and rotating systems, it is assumed that modal features remain more or less constant in case of presence of a mechanical fault, 
for example, pitting in bearings. This mentioned assumption is the basis for some of the recent and nonstationary signal processing techniques [6]. By eliminating the estimated structural response characteristics, a less sensitive signal to speed and load changes can be obtained [7]. These methods are based on removing the estimated vibration transfer path effects and modal characteristics of the system from measured signals. Note that the dynamical analysis of a structure/machine can be performed using the well-known Experimental Modal Analysis (EMA) techniques to extract the exact vibration transfer path in its frequency domain. In this regard, dynamic analysis can also be performed using continuous (permanent) measurements. For example, Operational Modal Analysis (OMA), as one of the most common techniques for dynamic analyses of running machines, is used for CM of wind turbines, for example, for tower and blades $[8,9]$, where some important modal info and parameters can be extracted, tracked, and analyzed for a specific application.

In addition to the traditional use of modal testing for design, Noise Vibration Harshness (NVH), and fault localization purposes, the Frequency Response Function (FRF) in modal analysis, as a vibration transfer path function, can be used in other applications such as fault isolation and ideal sensor placement. However, it should be pointed out that the modal analysis on a real structure/machine may not be feasible due to test environment restrictions or due to the lack of reliable mount data. It can therefore be shown that, with a reliable numerical model in hand, the corresponding dynamical analysis can be carried out numerically and reliably. The Multibody Simulation (MBS) approach has approved its usability in identification of system dynamic (modal) characteristics, as a reliable alternative for the real machine [10]. The MBS models can be used for assessments of load interactions between various components in a mechanical system [11]. These models can also represent a testing manner [12], in case there are some restrictions to performing measurements on the real machine. Recently, it is shown that the online or up-to-minute condition monitoring is also a growing application field for the so-called Digital Twin concept using MBS modelling $[13,14]$. Only relying on sensor data is sometimes not sufficient. In this situation the simulation (i.e., MBS) models, provided by the Digital Twin, can regularly be modified through measured data and reused after these modifications. By using the simulation models, it is also possible to interpret the measurements in a different way, rather than just detecting deviations from the norm.

For improving the performance of the machine monitoring and fault detection procedures, it can be shown that, as a complementary investigation in the scope of ideal sensing positions as well as estimation of the fault location, a modeloriented approach using MBS modelling may be a useful strategy. In addition to this, the model-oriented designing approach for CM and fault diagnosis algorithms may offer a new degree-of-freedom, in particular in the context of Blind Source Separation (BSS) family methods. The core of BSSbased signal processing method may be optimized according to insights from a comprehensive MBS model, where the excitation sources can be specified numerically and reliably. This becomes even more critical for those systems having many subsystems (i.e., components) running in varying operating conditions.

Rolling bearings, as one of the most common and important components in many mechanical engineering applications (e.g., drivetrains), are always considered as a part with potentially high-risk against the failures. Bearings have very significant impact on the global vibrations of a rotary mechanical system. Furthermore, in electrified vehicles, a bearing failure may lead to increasing the rotational friction of the rotor, and thus a decrease in the performance of the electric motor. Therefore, vibration-based fault detection and diagnosis of the rolling bearings have become a vigorous area of work and have attracted more and more attention in the literature $[15,16]$. These studies in condition monitoring are mainly focused on finding the best signal processing technique to detect an incipient failure. Since rolling element bearings are subjected to moving distributed radial and axial loads, any probable fault can excite the system resonances in a specific frequency range depending on neighboring components. It can be concluded that the captured bearing vibration signals carry the information about the system structure in a comparatively high frequency range, which can be interpreted as the transmission path(s) of the vibration. It is also known that the inherent frequency of a bearing is fixed resonance frequency/frequencies even if the fault repetition frequencies (bearing fundamental frequencies) tend to change with shaft speed. This can be a key factor in localizing the similar faulty bearing(s) rotating at the same speed.

Many research studies are dedicated to the field of vibration-based condition monitoring (CM), diagnosis, and fault detection, mostly in oil and gas, wind energy, and railway, including tracks and vehicles, sectors, yet fewer ones are focused on specific requirements and demands for a similar system in the field of automotive engineering. Although numerous general-purpose fault detection and diagnosis algorithms and methods using signal processing techniques are reported in the literature, implementation, applicability, reliability, and ideal (optimal) sensing positions for both offline and online CM systems are still a challenge due to the involved computational complexity and expensive hardware. On-Board Diagnostic (OBD) systems in automotive engineering applications are recently employed and used for various purposes (e.g., $\mathrm{Co}_{2}$ emission and electronic components). In order to achieve a reliable and cost-effective an OBD CM and fault detection system for the drivetrain of a vehicle, the diagnostic algorithm should be simple, robust, and beneficial from ideally located sensors. Since thousands of data points are captured and need to be processed during the monitoring process, characteristics of the captured signals, number and locations of the sensor(s), and the detection algorithm itself have become critical parameters for a precision monitoring.

The present study therefore aims to address and investigate some challenges and complexities in vibration-based fault detection and diagnosis for rolling bearings in automotive sector for a drivetrain of an electrified vehicle. In line with this purpose, an experimentally validated MBS model is used for the investigations. Note that a large number of manufacturers, in particular in automotive and wind energy 
sectors, already have and use numerical models for $\mathrm{NVH}$ and testing purposes, so that the modelling step in the development and design procedure of a dedicated and/or implemented condition monitoring systems would not be a time consuming step in designing the next generation monitoring and testing systems. It is worth stating here that this study should be considered as a basis for future works in combination of the fields vibration-based fault detection and numerical modelling.

In the present study, the use of structural dynamics in condition monitoring and fault detection of the complex mechanical systems are studied. The MBS model of a drivetrain for an electrified vehicle created and validated in a previous study is used in numerical analyses as a case study. First, a comprehensive theoretical basis of the vibration-based fault detection for rolling bearings is given. Then, a summary of the modelling strategy as well as subsequent revisions applied to the model to create faulty components (i.e., bearings) is described. Three artificially created faulty cases using the MBS model are then used for numerical investigations on bearing fault diagnosis. In further discussions, the role of Frequency Response Function (FRF), as the transfer path function, in faulty bearing(s) localization and also in finding the ideal sensor location is studied. Finally, some concluding remarks are made about the reliability and applicability of the approach used in this study for bearing fault diagnosis and sensor placement.

\section{Vibration-Based Rolling Bearing Fault Diagnosis: Theoretical Background}

In general, a mechanical system's dynamic characteristics (i.e., vibration response) are specified by the corresponding transfer path and excitation sources, as governed by the mathematics of convolution. According to the previous findings in the literature [2], a typical captured vibration signal by a sensor, $x(t)$, of a machine consists of shaft(s), electric motor, rolling bearing(s), gear stage(s), coupling(s), and so on in its operating condition with an individual focus on rolling bearing vibrations can be considered as

$$
x(t)=h(t) *\left[s_{1}(t)+s_{2}(t) y(t)+n(t)\right]
$$

where $y(t), h(t)$, and $n(t)$ correspond to the bearing, the transfer path which modulates the fault(s) response to a higher frequency range (i.e., system resonances), and noise originating from measurement system, numerical errors, and/or incidental and transient excitations, and so on, respectively. While both $s_{1}(t)$ and $s_{2}(t)$ are related to uncontaminated periodic responses of all other machine parts (due to electric motor, gears, shaft misalignment and unbalances, etc.), $s_{1}(t)$ represents the unmodulated part and $s_{2}(t)$ represents the modulated part of periodic responses with the bearing signal. Also, * stands for the convolution operator. Eq. (1) can then be extended as

$$
x(t)=h(t) *\left[s_{1}(t)\right.
$$

$$
\left.+s_{2}(t)\left(\sum_{n=1}^{N} \sum_{k=-\infty}^{\infty} a_{n}(t) \delta\left(t-k T_{n}\right)\right)+n(t)\right] \text {, }
$$

where $n, a_{n}(t)$, and $\delta(t)$, respectively, are the number of fault(s) existing in the bearing (varying from 1 up to $N=4$, as there are generally 4 types of faults in a rolling bearing), the amplitude of the vibration response to the $n$th fault which reflects the strength of the fault, and Dirac delta function, where $T_{n}$ denotes the time period between impulses (i.e., $1 / \mathrm{BPFO}$ or $1 / \mathrm{BPFI})$. Note that the maximum amplitude of $a_{n}(t)$ can be assumed to be constant over time for outer race defects due to its stationary condition [17]. The amplitude spectrum of the vibration signal model in (2) for a system having a bearing with a single fault can therefore be derived using Fourier Transform as

$$
\begin{aligned}
& \mathscr{F}\{x(t)\}=X(\omega)=\mathscr{F}\{h(t) \\
& \left.\quad *\left[s_{1}(t)+s_{2}(t)\left(\sum_{k=-\infty}^{\infty} a(t) \delta(t-k T)\right)+n(t)\right]\right\} .
\end{aligned}
$$

In case the signal energy distribution is desired, the energy spectral density of $\omega$ can also be obtained as

$$
|X(\omega)|^{2}=X(\omega) \overline{X(\omega)}
$$

where $\overline{X(\omega)}$ denotes the complex conjugate of $X(\omega)$.

It is well-known that the amplitude spectrum of vibration signals may not detect and display the bearing fault frequencies. In such cases, envelope spectrum is the most common method for the fault frequency detection. Nevertheless, in harsh operating conditions, the calculated envelope spectrums are not capable of displaying the fault diagnostic information (i.e., bearing fault frequencies) due to heavy background noise and/or existence other excitation sources. As a result, some preprocessing steps, prior to the envelope analysis, are strongly recommended in the literature.

Recall from convolution properties

$$
\mathscr{F}\{(f * g)(t)\}=F(\omega) G(\omega)
$$

and Nyquist sampling theorem

$$
\mathscr{F}\left\{f(t) \sum_{k=-\infty}^{\infty} \delta\left(t-k T_{r}\right)\right\}=\frac{1}{T_{r}} \sum_{k=-\infty}^{\infty} F\left(\omega-k \omega_{r}\right) .
$$

Using the convolution and Nyquist sampling properties, the right-hand side of (3) can be expanded as follows:

$$
\begin{aligned}
X(\omega)= & \mathscr{F}\left\{h(t) * s_{1}(t)\right\} \\
& +\mathscr{F}\left\{h(t) * s_{1}(t)\left(\sum_{k=-\infty}^{\infty} a(t) \delta(t-k T)\right)\right\} \\
& +\mathscr{F}\{h(t) * n(t)\} .
\end{aligned}
$$


Then,

$$
\begin{aligned}
X(\omega)= & \underbrace{H(\omega) S_{1}(\omega)}_{\begin{array}{c}
\text { non-related } \\
\text { uncontaminated } \\
\text { periodic } \\
\text { components } \\
\text { (e.g. gear stages) }
\end{array}} \\
& \underbrace{H(\omega) S_{2}(\omega)\left(\frac{1}{T} \sum_{k=-\infty}^{\infty} A(\omega-k \omega)\right)}_{\begin{array}{c}
\text { Modulated impulsive } \\
\text { components, which } \\
\text { contain bearing diagnostic } \\
\text { information, } A(\omega-k \omega)
\end{array}} \\
& +\underbrace{H(\omega) N(\omega)}_{\begin{array}{c}
\text { Noise } \\
\text { representing random and } \\
\text { transient components }
\end{array}} .
\end{aligned}
$$

Eq. (8) reveals that $X(\omega)$ contains various and crucial information regarding current status of the test machine (i.e., bearing system). These three constituents can be categorized as (I) uncontaminated period components, (II) modulated impulsive components, and (III) noise. From rolling bearing fault detection point of view, the diagnostic info is associated with the term $H(\omega) S_{2}(\omega) y(\omega)$.

A wide range of studies available in the literature are dedicated to signals containing multiple sources $[6,18]$. Source separation techniques are proposed in order to separate and eliminate (if needed) the nonrelated periodic components. Perhaps the most common prewhitening technique for eliminating the nonrelated periodic components in rolling bearing vibration signals is autoregressive (AR) filtering, documented and suggested in [2]. Prior to further postprocessing, the other component needs to be eliminated is the noise, $H(\omega) N(\omega)$, representing the random and unwanted signals. This can be carried out using denoising algorithms, such as SVD-based denoising method presented in [19]. As a result, after the prewhitening and denoising processes, the denoised version of $X(\omega)$, called $X_{d}(\omega)$, can be estimated as

$$
\begin{aligned}
X_{d}(\omega) & =H(\omega) S_{2}(\omega) y(\omega) \\
& =H(\omega) S_{2}(\omega)\left(\frac{1}{T} \sum_{k=-\infty}^{\infty} A(\omega-k \omega)\right) .
\end{aligned}
$$

In order to extract the modulating component, $A(\omega-$ $k \omega)$, in (9), demodulation should be performed. For this, an appropriate estimation of $H(\omega)$ is required in order to extract the fault diagnostic info. One method to estimate $H(\omega)$ in the desired frequency range (i.e., system resonance) is the spectral kurtosis (SK) [20]. Using the same property of convolution theorem given earlier, demodulating in the frequency domain can then be formulated as

$$
\begin{aligned}
& S_{2}(\omega) A(\omega-k \omega) \\
& \quad \approx \text { lowpass filter }\left\{\widehat{\mathrm{H}(\omega)} * S_{2}(\omega) y(\omega)\right\},
\end{aligned}
$$

where $\widehat{H(\omega)}$ is an estimation of $H(\omega)$. Again, here $S_{2}$ represents, for example, shaft-related components originating from unbalances, torsional vibrations, and eccentric faults. In some cases, in particular in complex mechanical systems, estimation of $H(\omega)$ can be a complex and time consuming step.

Back to the present theoretical formulation in (9), in practice, however, demodulation on the bearing signal, $y(t)$, is carried out by a bandpass filtering (e.g., SK-based optimized filter) with a central-frequency at the system resonance, and then Hilbert Transform in the time domain as

$$
A(t)=\sqrt{y^{2}(t)+i y_{H}^{2}(t)},
$$

where $A(t)$ is also known as the envelope signal and $y_{H}$ is the Hilbert Transform of $y(t)$ as

$$
y_{H}(t)=\frac{1}{\pi} \int_{-\infty}^{\infty} y(t) \frac{1}{(t-\tau)} d \tau .
$$

The so-called envelope spectrum is then be obtained by Fourier Transform of the envelope signal, $A(t)$, as

$$
\operatorname{Env}\{y(t)\}=\mathscr{F}\{A(t)\} .
$$

As is being shown, the transfer path function, $H(\omega)$, plays a major role in diagnostic vibration signals captured from a mechanical system. In the following sections in the present study it is shown that the transfer path can be analyzed in more detail in order to estimate fault location and ideal sensing positions. This function can be represented by Frequency Response Function.

\section{Summary of Numerical Modelling}

In this section, a brief summary of numerical modelling is presented. In addition, some details of the model revision for fault detection purposes are described.

In general, the dynamical systems can be presented in terms of their equation of motion (E.o.M.) as

$$
[M]\{\ddot{u}\}+[C]\{\dot{u}\}+[K]\{u\}=\{f\},
$$

where $\{u\},\{f\},[M],[C]$, and $[K]$ stand for the displacement vector, the force vector (including external and internal torques and moments), inertia (mass), damping, and stiffness matrices of the involved bodies, respectively. In Elastic Multibody Simulation (EMBS), the E.o.M. may be solved in an iterative way, either in time domain or frequency domain. For this, the system matrices and the force (e.g., containing the excitations) vector need to be formed first. However, prior to assemblies of the machine parts by implementation of the masses, stiffness, and the external excitations, EMA is employed in order to validate the modal properties of each elastic parts used in the model. Following this bottomup process, the entire dynamical system (i.e., drivetrain) consisting of all validated subsystems is validated against the experiments. Some details of the numerical modelling and validation process are readily available in [21]. The previously created EMBS model of a test Battery Electric Vehicle (BEV) for which the numerical investigations are performed is shown in Figure 1. 


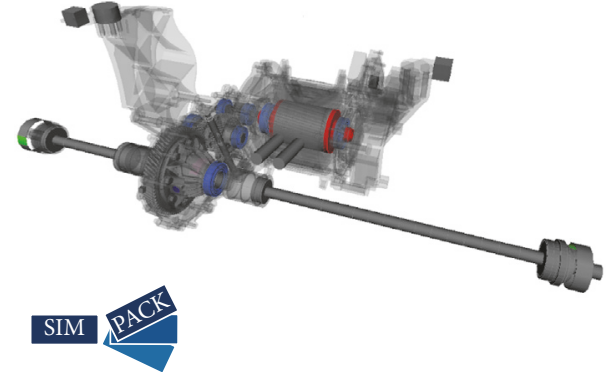

FIGURE 1: The validated MBS model of an electrified vehicle's drivetrain.

In line with the purpose of rolling bearing fault diagnosis, the numerical model investigated and validated in the previous paper is used in numerical analysis. As discussed in the previous section, in rolling bearings with localized defects, an excitation force may be produced when the rolling elements move over the faulty area [22]. This force may then results in sudden and sharp changes in vibration trend of the system, that is, $y(t)$ in (1), accordingly. The magnitude and the duration of this force are related to the radial load carried by the rolling element, the defect natures (severity and age), velocity, and the material properties used in the bearing [23]. Assuming a dependency between the excitation force due to the localized bearing defect and its generated vibrations, the bearing faults are modelled in terms of their excitation forces by amplifying the magnitudes of the corresponding force element in a certain zone in the force vector in (14). A computer program developed in Fortran Programming Language is used to generate the excitation force, considering the bearing kinematics. Again, the magnitude of the excitation force depends on various parameters, and this is beyond the scopes of the present study. Some various shapes and magnitudes, however, are generated and used in numerical investigations. The developed computer program is capable of locating the given number of defective bearings at the given positions and creates the dynamic excitation forces suitable for calculations (i.e., time integration) in a Multibody Simulation platform, namely, SIMPACK. As the rolling elements pass through the faulty area in the bearing, duration of each impact by interaction between rolling elements and faulty area highly depends on the shaft speed. In general, the impulse-like force, $f_{e}(t)$, generated due to repetitive interactions of the rolling elements with the defective area, can be expressed as

$$
f_{e}(t)=A(t) \sum_{k=-\infty}^{\infty} \delta(t-k T)
$$

where $A(t)$ and $T$ are magnitude of the force and time period between impulses (i.e., 1/BPFO), respectively. Note that $T$ may also be considered as a time-varying parameter for nonstationary operating conditions (i.e., nonconstant shaft velocity). It is then assumed that the desired excitation force depends on the impulse-like force obtained in (15), and the radial load, $P(\theta)$. The excitation force in the radial

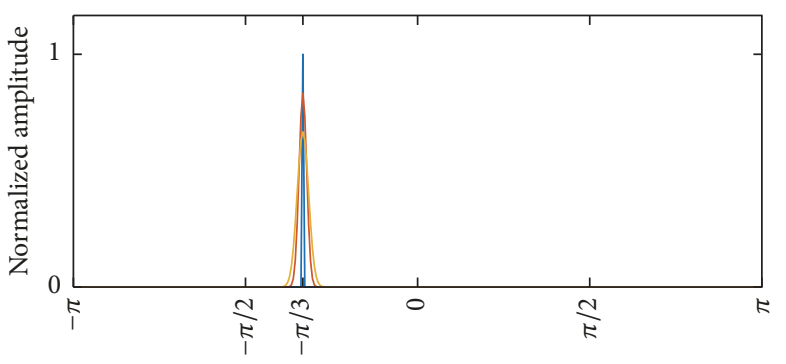

Angle (rad)

$$
\begin{array}{r}
f_{e 1}(\theta) \\
-f_{e 2}(\theta) \\
-f_{e 3}(\theta)
\end{array}
$$$$
\text { Impulse-like }
$$$$
\text { excitation function }
$$

FIgURE 2: Illustration of three distinct impulse-like forces for the single localized bearing fault with respect to angular position of the rolling element.

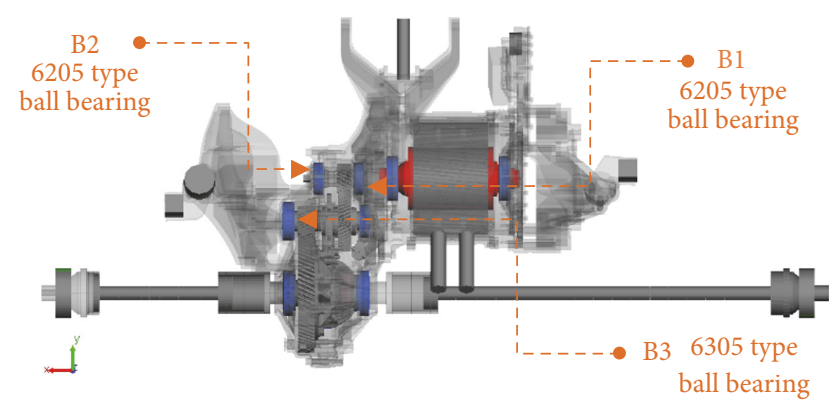

FIGURE 3: Illustration of the faulty test bearings, namely, B1, B2, and B3.

direction, $f_{b}(t)$, representing the fault, can be therefore obtained as

$$
f_{b}(t)=f_{e}(t) P(t) \cos \theta
$$

where $P(t)$ and $\theta$ are the radial load on the rolling element/raceway and rolling element position, respectively. Figure 2 shows three distinct forces artificially generated and applied to the test bearings in the MBS model. In the present study, it is assumed that $f_{e 1}, f_{e 2}$, and $f_{e 3}$ represent three different fault stages on outer race as a tiny crack (e.g., initial stage), medium-size fault (e.g., intermediate-stage), and a large-size fault (e.g., advanced stage), respectively.

Three deep groove ball bearings, two of which are 6205 type and one is 6305 type, are used as the test bearings. The test bearings are labeled and shown in Figure 3.

Table 1 lists the corresponding geometric details of ball bearings used in the tests. The fundamental fault frequency for outer race, $\mathrm{BPFO}$, where outer race is stationary and inner race rotates in shaft can be formulated as

$$
\mathrm{BPFO}=\frac{N_{B}}{2} f_{r}\left(1-\frac{D_{b} \cos \beta}{D_{p}}\right),
$$

where are $N_{B}, f_{r}, D_{b}, D_{p}$, and $\beta$ number of rolling elements, shaft rotating frequency, rolling element diameter, pitch diameter, and rolling bearing contact angle, respectively. 
TABLE 1: The geometry details of test bearings.

\begin{tabular}{lccccc}
\hline Bearing & Bearing type & Pitch diameter & Ball diameter & Number of balls & Contact angle \\
\hline B1 & 6205 type & $38.9 \mathrm{~mm}$ & $7.9 \mathrm{~mm}$ & 9 & $0^{\circ}$ \\
B2 & 6205 type & $38.9 \mathrm{~mm}$ & $7.9 \mathrm{~mm}$ & 9 & $0^{\circ}$ \\
B3 & 6305 type & $43.4 \mathrm{~mm}$ & $11.1 \mathrm{~mm}$ & 7 & $0^{\circ}$ \\
\hline
\end{tabular}

TABLE 2: Details of the numerical case studies.

\begin{tabular}{|c|c|c|c|c|}
\hline Case study & Test bearing(s) & Fault type & Fault freq. $\mathrm{Hz}$ & Fault freq. ord \\
\hline$\# 1$ & B1 & $\begin{array}{l}\text { Outer race } \\
\text { Exc.: } f_{e 1}\end{array}$ & $32[\mathrm{~Hz}]$ & 3.551 \\
\hline \multirow[b]{2}{*}{$\# 2$} & B1 & $\begin{array}{l}\text { Outer race } \\
\text { Exc.: } f_{e 1}\end{array}$ & $32[\mathrm{~Hz}]$ & 3.551 \\
\hline & B3 & $\begin{array}{l}\text { Outer race } \\
\text { Exc.: } f_{e 1}\end{array}$ & $11[\mathrm{~Hz}]$ & 2.61 \\
\hline \multirow[b]{2}{*}{ \#3 } & B1 & $\begin{array}{l}\text { Outer race } \\
\text { Exc.: } f_{e 1}\end{array}$ & $32[\mathrm{~Hz}]$ & 3.551 \\
\hline & B2 & $\begin{array}{c}\text { Outer race } \\
\text { Exc.: } f_{e 1}\end{array}$ & $32[\mathrm{~Hz}]$ & 3.551 \\
\hline
\end{tabular}

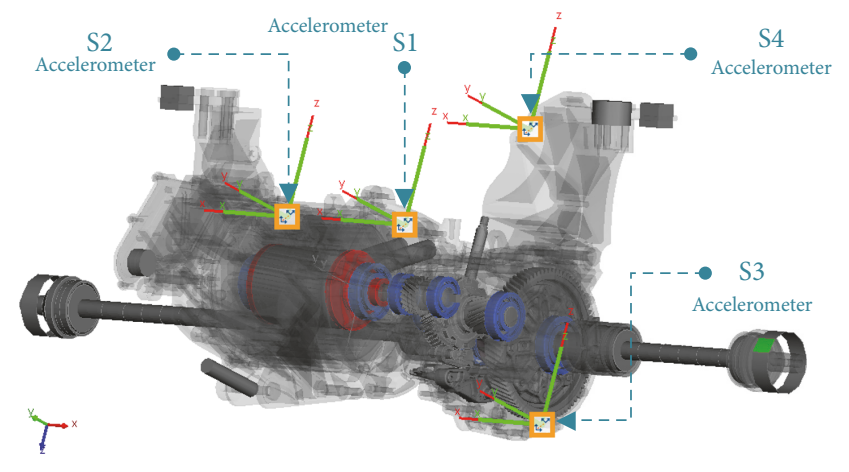

FIGURE 4: Illustration of the sensor positions, namely, S1, S2, S3, and S4.

In addition, four sensor locations are specified and shown in Figure 4. Note that sensors measure acceleration in normal to surface direction. The related fault frequencies for the test bearings are obtained and presented in Table 2. Three case studies, namely, single faulty bearing, multiple faulty bearings rotating in different velocities (different shafts), and multiple faulty bearings rotating in the same velocity (same shaft), used in numerical investigations, are also listed and detailed in Table 2. The fault stages for all the case studies are assumed as a tiny crack (i.e., $f_{e 1}$ ) on outer races. The other fault stages (i.e., $f_{e 2}$ and $f_{e 3}$ ) have been investigated in the section further discussions in some detail.

The acceleration signals of the MBS model in normal to surface direction are captured as fault signatures. The data are collected for $2 \mathrm{~s}$ at $20 \mathrm{kHz}$ rate in SIMPACK. In all the simulations, both drive and intermediate-stage shafts are given fixed nominal angular velocities in the steady-state area which are shown in Figure 5. As seen, both shafts are subjected to some torsional vibrations due to fluctuations in torque loads from electric motor. The simulation results are exported to MATLAB for postprocessing. Note that only the second half of the simulations, that is, from $1 \mathrm{~s}$ to $2 \mathrm{~s}$, are used for postprocessing to avoid high amount of nonstationarities during run-up.

It is also worth mentioning that in order to avoid from unnecessary complexities, the gears mesh stiffness values are assumed to be constant over time. As a result, the term $H(\omega) S_{1}(\omega)$ in (8) has not been taken into account in the present study.

\section{Results}

In this section, the captured vibration signals of simulations are analyzed and investigated in both time and the frequency domains. The aim is to show and discuss the advantages, disadvantages, and limitations of the well-known envelope analysis in bearings fault detection and diagnosis. In what follows, the details of these simulations are presented for three different case studies.

4.1. Case Study 1: Single Faulty Bearing. This case study investigates the dynamical behavior of the MBS model having one single faulty bearing on the drive shaft. Figure 6 illustrates the artificially generated excitation force applied to B1 in time domain and its representation in frequency domain. As stated earlier, this excitation force is generated using the excitation function, $f_{e 1}$, to analyze the vibration behavior of the model, as well as the real drivetrain, in a condition with a bearing having an initial localized fault such as a tiny crack. The magnitude of force acting on the outer race of $\mathrm{B} 1$ with a certain repetition frequency (i.e., BPFO) is assumed to be $120 \mathrm{~N}$ in this case study.

Figure 7 shows the signals captured from four different locations in the time domain as well as their spectrums up 


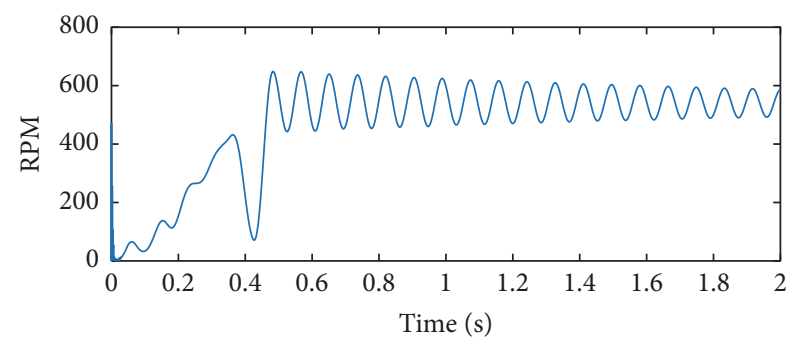

(a)

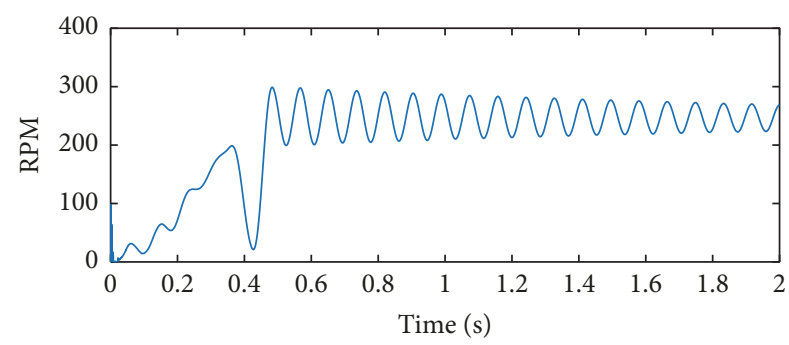

(b)

Figure 5: Angular velocities of (a) drive shaft (b) intermediate-stage shaft.

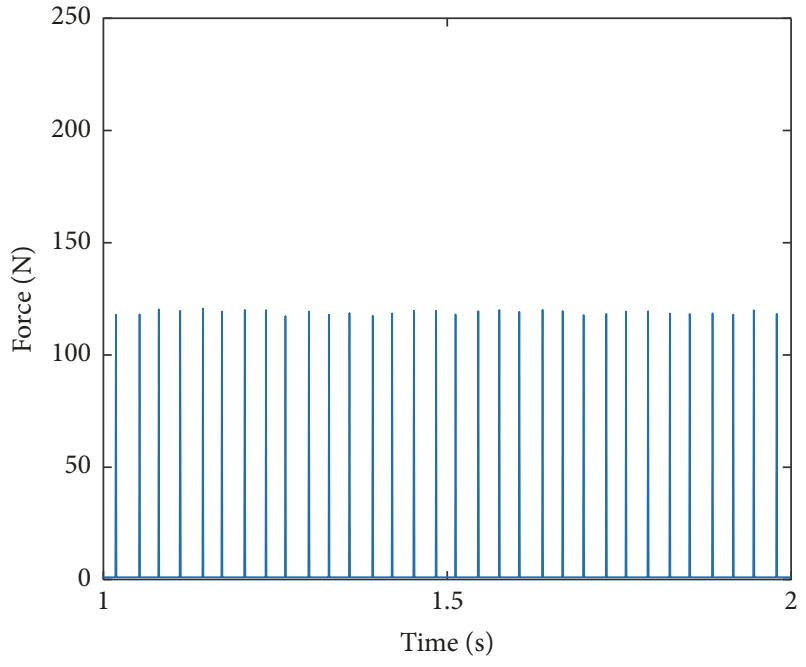

(a)

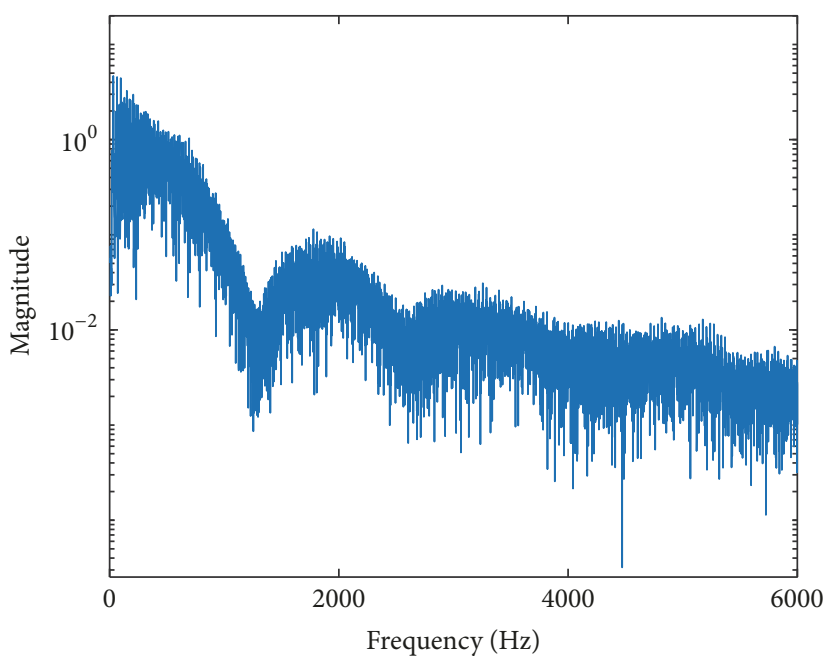

(b)

FIGURE 6: Illustration of the applied excitation force to B1 in (a) time domain and (b) frequency domain.

to the Nyquist frequency. As expected, the excitation force excites the system resonances in a specific frequency range. However, by analyzing the force spectrum in Figure 6(b) and the obtained spectrums in Figure 7, it is revealed that small amount of energy in a high frequency range strongly excites the system resonances. This behavior may be explained by transfer path of the vibration from $\mathrm{B} 1$ to the sensing locations. In the next section, it is shown that using this valuable insight, an ideal sensor location (between four sensing locations) can be selected. Again, the results presented here suggest that S3 can yield better diagnostic results in terms of the amplitude of faulty signal.

The corresponding envelope spectrums of time domain signals in Figure 7 are illustrated, respectively, in Figure 8. Since the variations in shaft velocities are very low, that is, shown in Figure 5, the smearing effect in envelope spectrums is negligible. However, as expected, the fault frequencies are highly modulated with torsional vibrations, and it is more obvious in signal captured from S3. It is seen that all envelope and envelope order spectrums can reveal the fault frequency. Note that the results presented in Figure 8 make these findings more obvious where the diagnostic information obtained in envelope spectrums is very sharp and obvious for S3, and the fault frequencies have small magnitudes in S1.

In this case, it is seen that the envelope analysis, briefly reviewed and explained in Section 2, is capable of detecting the fault frequency, taking into account the fact that the magnitudes are greatly influenced by related transfer path and system structure. This suggests that, using a proper FRF function, it is possible to select an ideal sensing location for monitoring and test purposes.

4.2. Case Study 2: Multiple Faulty Bearings on Different Shafts. The analyses carried out for the case study 1 are repeated here for the case study 2, but this time, as shown in Figure 9, two excitation forces using the excitation function, $f_{e 1}$, having magnitudes of $120 \mathrm{~N}$ and $200 \mathrm{~N}$ are applied, respectively, to B1 and B3. Again, from frequency domain point of view, both excitations carry a high level of energy in low frequency band, but low level of energy in high frequency range (i.e., higher than $1 \mathrm{kHz}$ ).

Figure 10 shows the captured vibration signals, which carry fault diagnostic information for two distinct bearings. The great influence of the sensor location on the fault detectability feature of the signals is very obvious. In fact, 


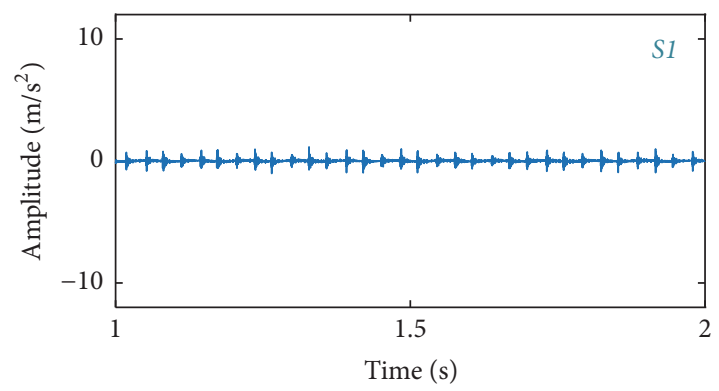

(a)

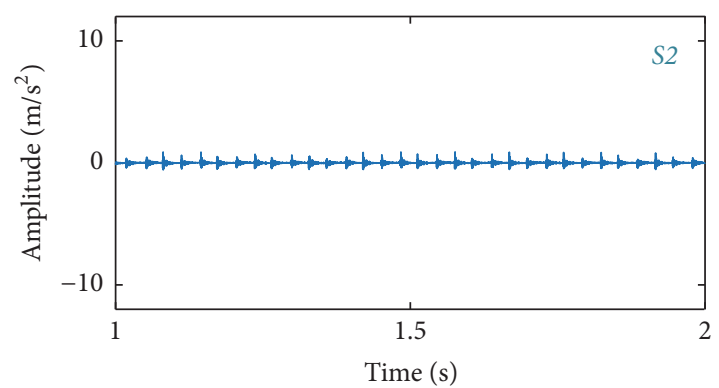

(b)

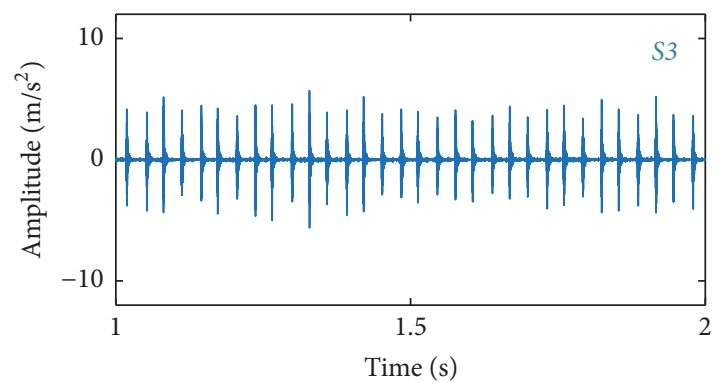

(c)

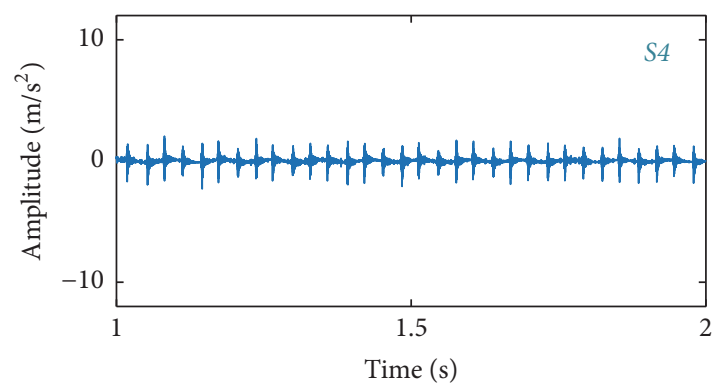

(d)

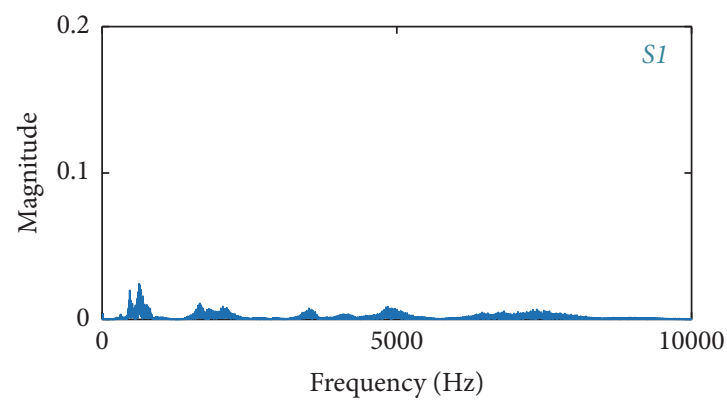

(e)

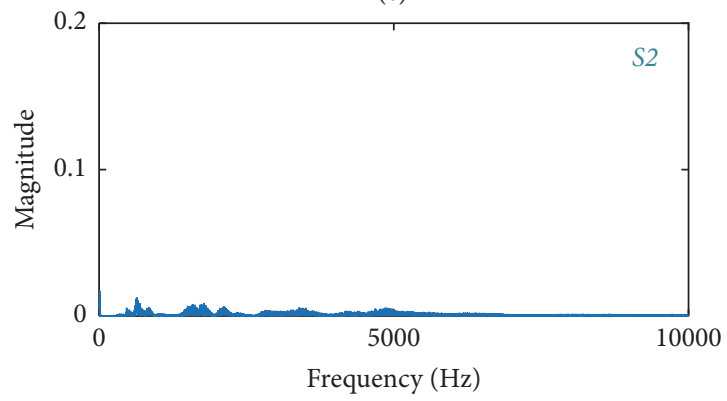

(f)

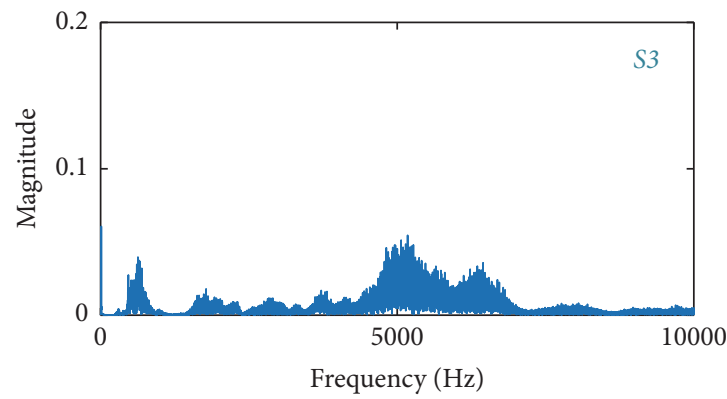

(g)

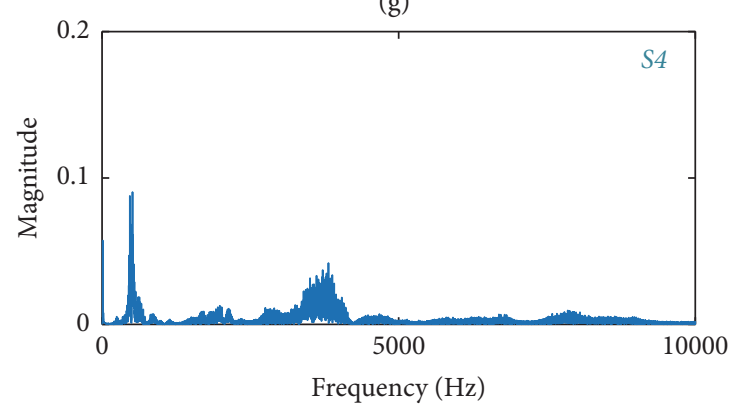

(h)

FIGURE 7: Case study 1: the captured time domain vibration signals from S1 to S4 in (a) to (d), respectively, and their corresponding spectrums in (e) to (h).

the amplitude of the impact trains, as the symptom of existence of bearing faults, in signal captured using S3 is approximately three times greater than that the one obtained using S4 and much sharper than other two sensors. This is an important result, showing that the performance of a CM system is also highly dependent on the sensor location.

The comments made before about the location of sensor on detecting feature of the envelope spectrum are also valid here. Once again, the highest magnitudes in the fault frequencies appear in the envelope spectrums obtained from S3. However, it is quite obvious that the diagnostic results for B3 are not as good as those of for B1.

Similar to the previous case study, the envelope order spectrums using the related rpm info are computed and presented in Figure 11. The resampling process from time domain to angular domain in order tracking can eliminate the modulation effect of torsional vibrations by removing 


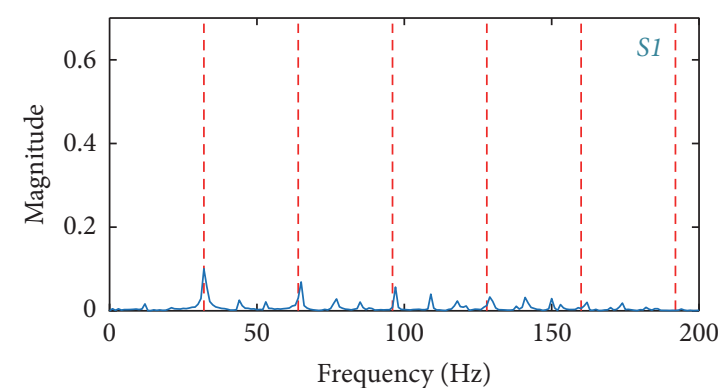

(a)

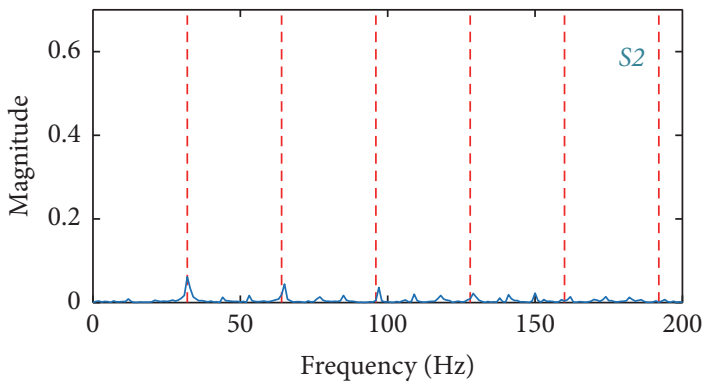

(b)

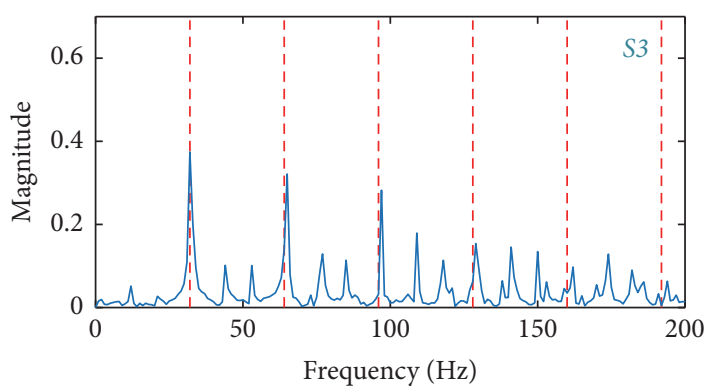

(c)

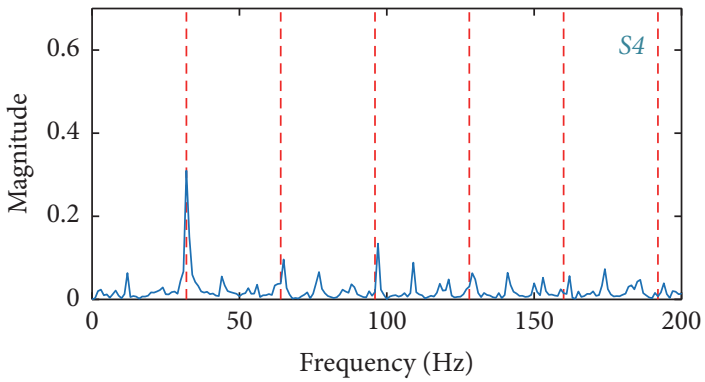

(d)

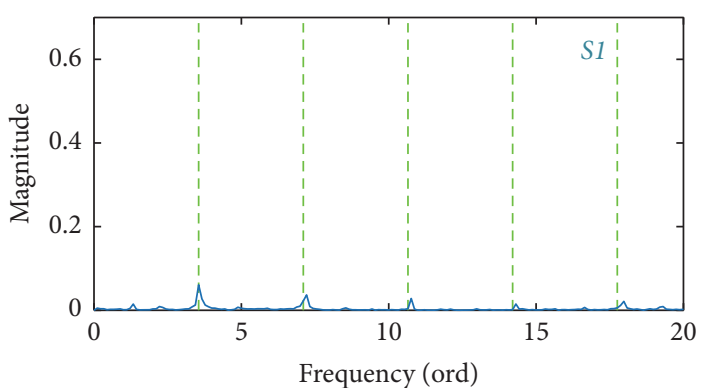

(e)

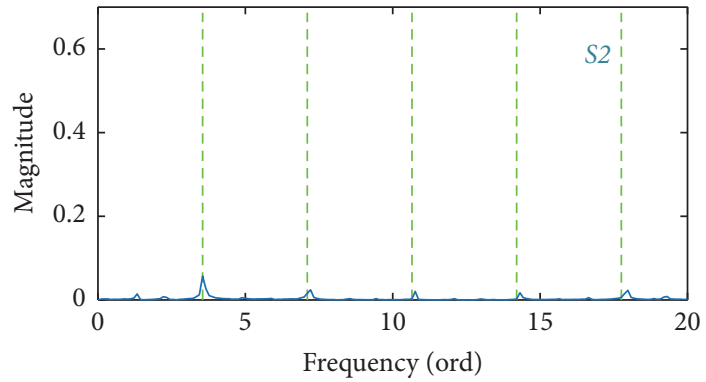

(f)

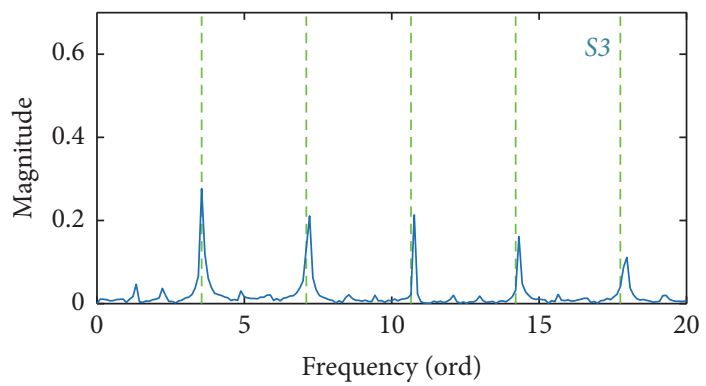

(g)

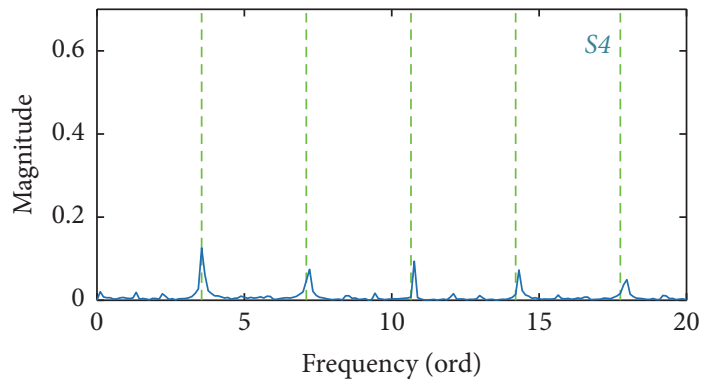

(h)

FIGURE 8: Envelope spectrums (a-d) and envelope order spectrums (e-h) for the time domain signals in Figure 7, respectively, from (a) to (d).

the side-bands around the fault frequency and its higher harmonics.

4.3. Case Study 3: Multiple Faulty Bearings on the Same Shaft. In previous case studies, the performance of the envelope analysis is assessed using two distinct bearings. It is shown that the envelope spectrum can yield reliable diagnostic results for cases having well separated fault frequencies. Here, again a case study using multiple faulty bearings, but on the same shaft, is investigated.

Figure 12 shows the force excitations applied to B1 and B2 having, respectively, magnitudes of $120 \mathrm{~N}$ and $80 \mathrm{~N}$. Also, Figure 13 shows the time and frequency domains of the vibration responses obtained from four different locations. Again, similar to the previous cases, in contrast to the applied low external energy level in high frequency range to the 


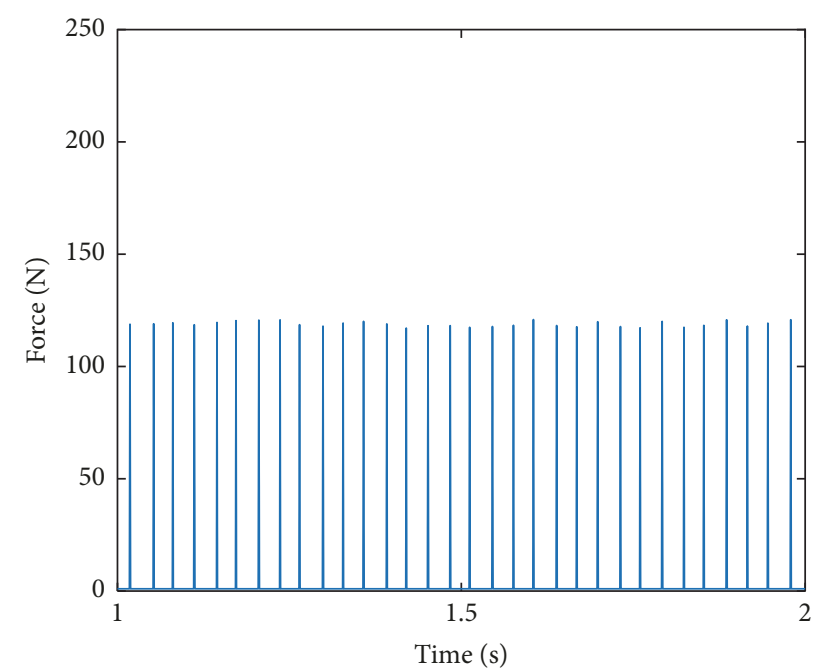

(a)

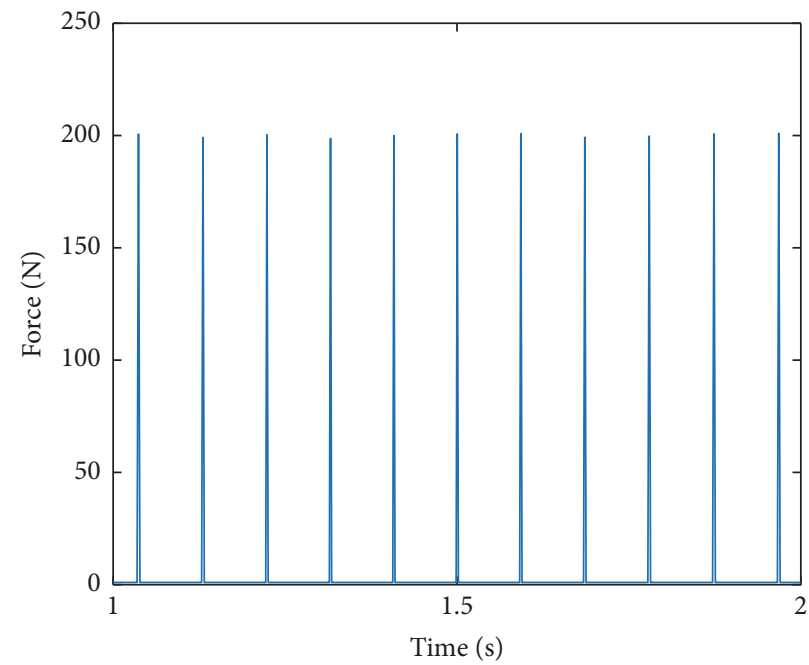

(b)

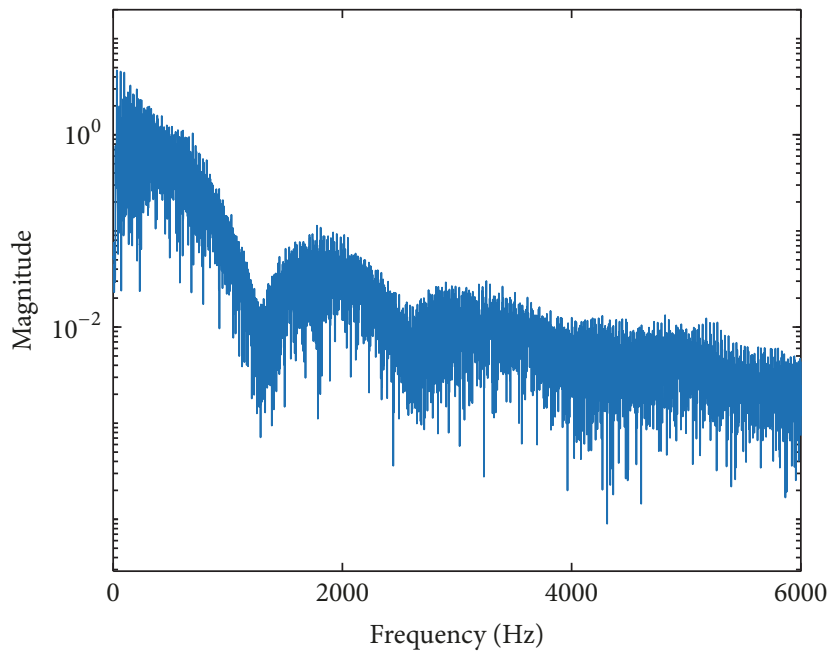

(c)

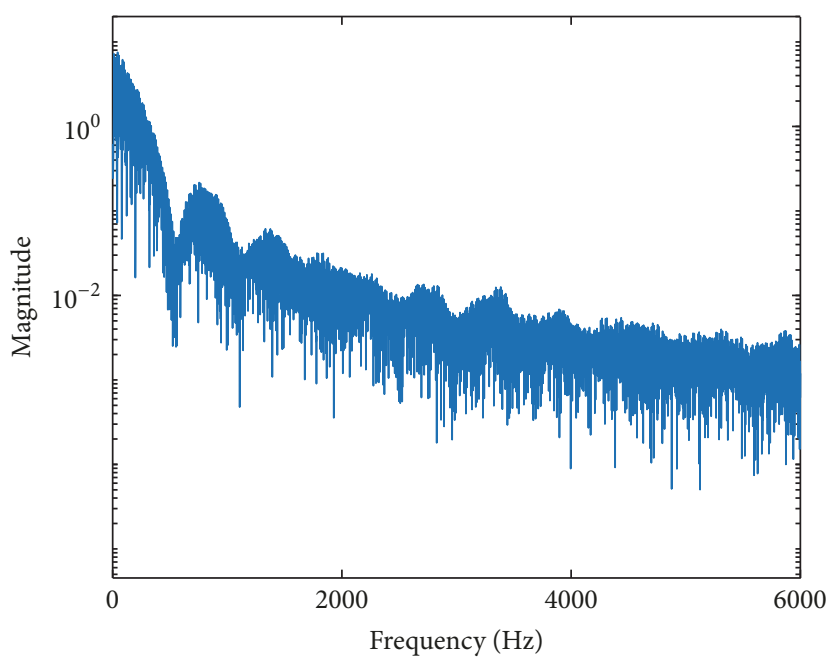

(d)

Figure 9: Illustration of the excitation forces to B1 and B3 in the time domain in (a) and (b), and in the frequency domain in (c) and (d), respectively.

system by these forces, inspection of the frequency spectrums in Figure 13 reveals that the system is more sensitive in higher frequencies due to its dynamical characteristics.

Figure 14 shows the envelope spectrums and envelope order spectrums of the time domain signals in Figure 13. As seen, due to overlying of the diagnostic information of $\mathrm{B} 1$ and B2, it is not possible to detect and diagnose both the bearings by relying merely on signal frequency domain information. This is an illustration of limitation of envelope spectrum in revealing that the fault frequencies of the same bearings rotate on the same rotating shaft. In the following section, in further discussion, it is shown that this can be explained by the combination of the structural dynamics and frequency domain signal processing.

According to the results calculated and shown in Figure 14, it is also can be seen that the signals captured using S3 and S4 share more or less same level of fault magnitudes. This can be explained by existence of a fault in B2, which excites the system in a different frequency range; thus $\mathrm{S} 4$ and $\mathrm{S} 3$ may have a nearly similar transfer path to the faulty area.

Again, the effects of torsional vibrations, as the term $S_{2}$ in (10), are very clear in terms of side-bands around the bearing fault frequency.

\section{Further Discussions}

5.1. Ideal Sensor Placement. As briefly discussed in introduction, in reality, the number of used sensors is limited due to various reasons, and this may result in some restrictions. As a result, optimization of the location of sensor(s) becomes a necessary and crucial step in practice for a given problem. The problem of determining the ideal or optimal sensor placements has already received much attention. Most of them share a common basis and deal with a specific application 


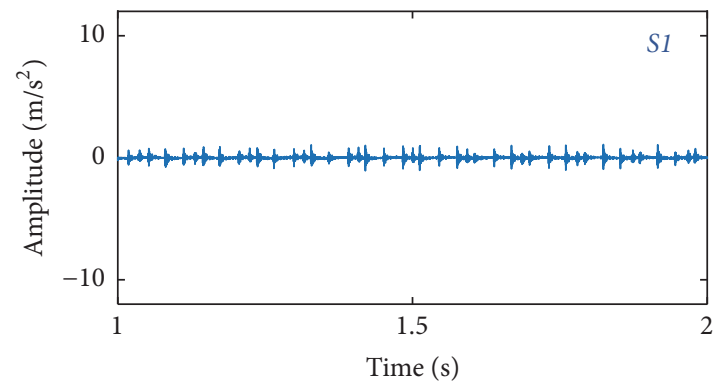

(a)

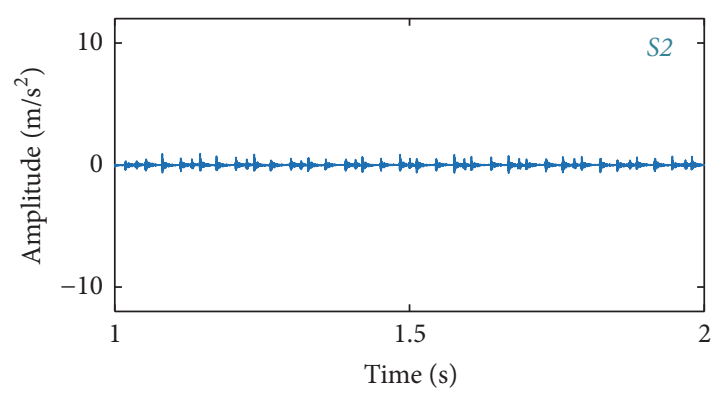

(b)

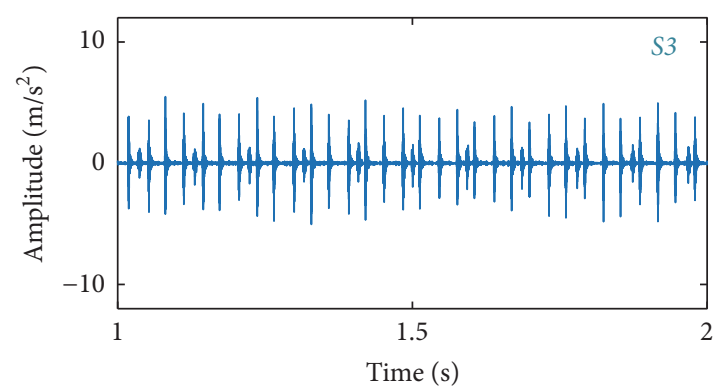

(c)

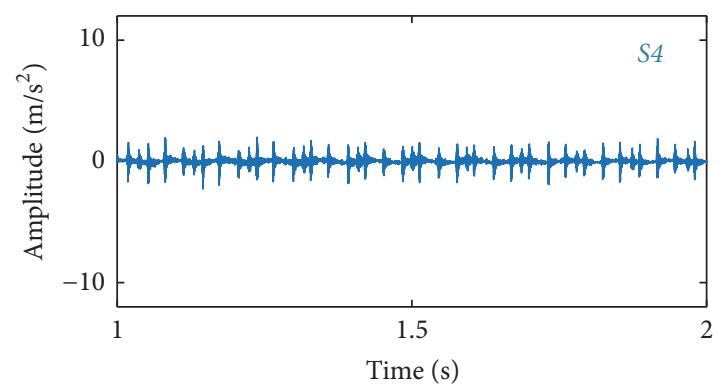

(d)

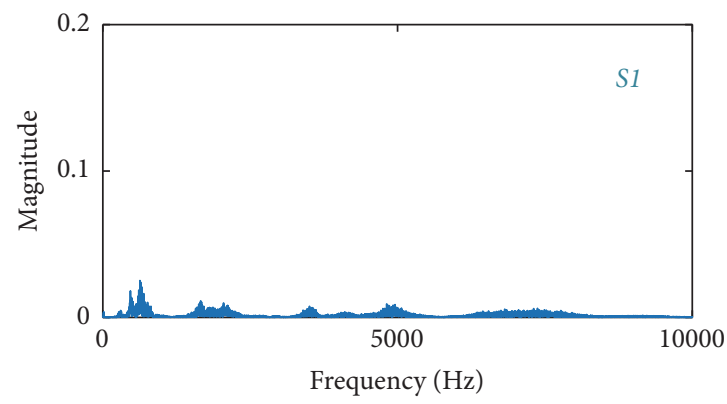

(e)

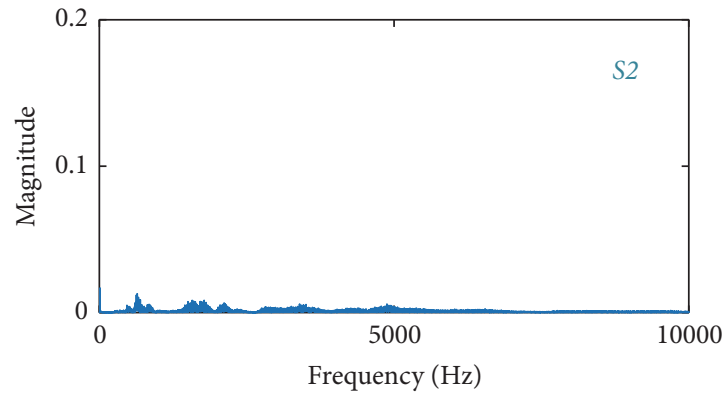

(f)

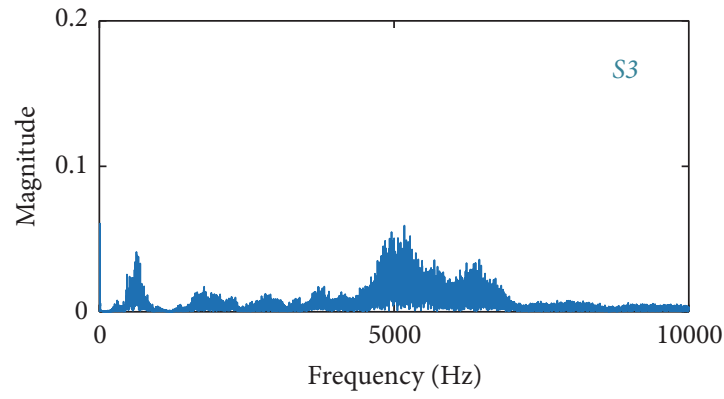

(g)

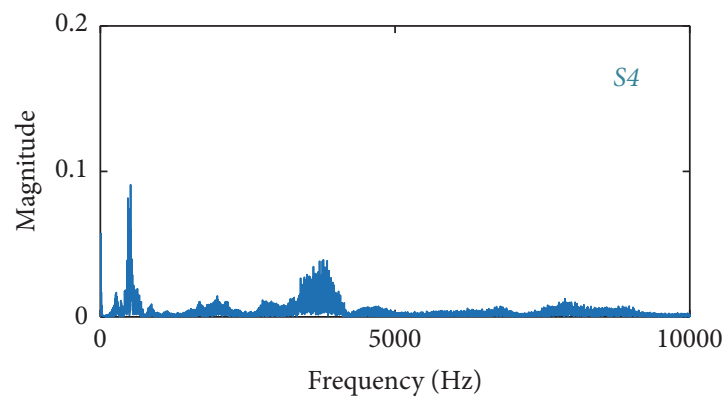

(h)

Figure 10: Case study 2: the captured time domain vibration signals from S1 to S4 in (a) to (d), respectively, and their corresponding spectrums in (e) to (h).

other than condition monitoring and fault detection such as modal testing and control engineering. According to the findings in the previous section, this section is concerned with the specific question of ideal sensor placement for the purpose of bearing fault detection. It should be noted that here, unlike a classical optimization problem, the sensing positions are compared and investigated in terms of their fault detectability.

As discussed and shown in theory, the transfer path of the bearing vibration signal plays a major role in the bearing fault detection and has its effects on the performance of a CM system. These effects can be analyzed in two different aspects: (I) the influence of transfer path on diagnostic information, and (II) estimation of transfer path in the desired frequency range for the bandpass filtering prior to envelope analysis.

Figure 15 illustrates the FRFs obtained from four different locations, namely, S1, S2, S3, and S4, based on two distinct excitation points, on B1 and B3. It is well-known that the FRFs can represent the transfer path in a mechanical system. 


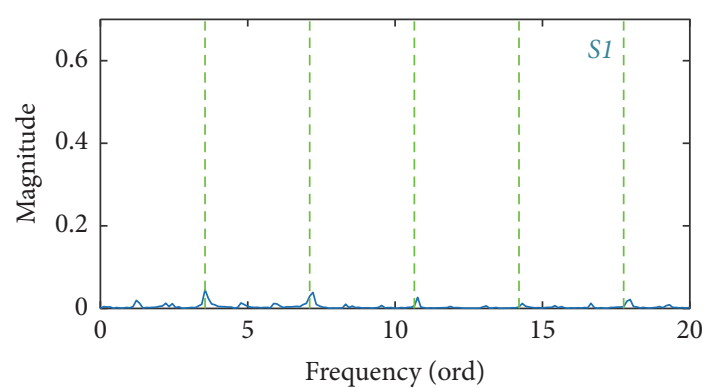

(a)

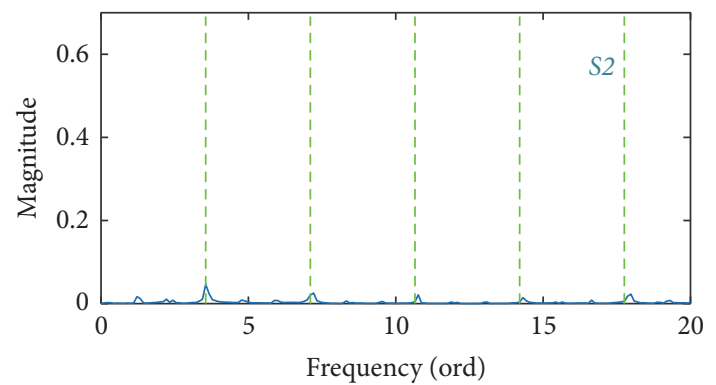

(b)

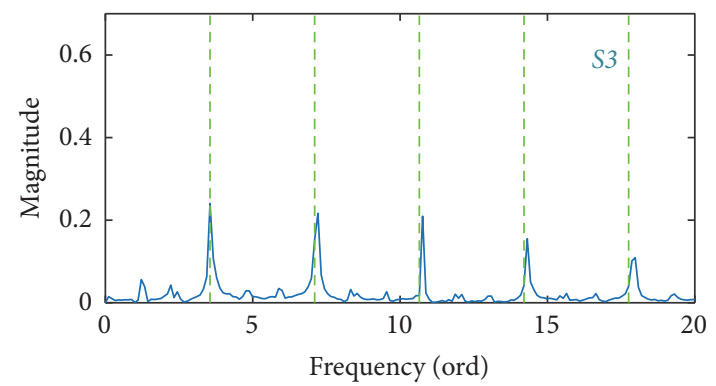

(c)

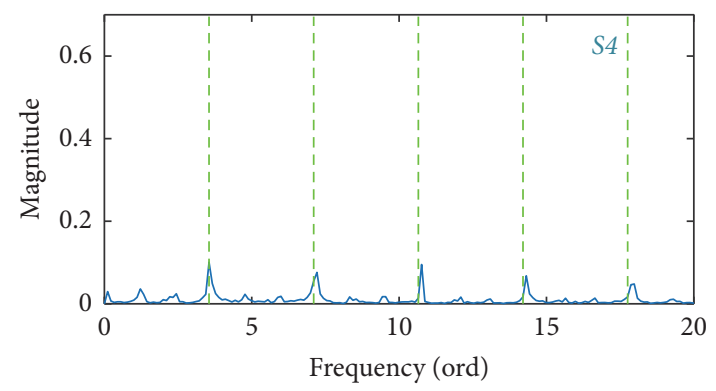

(d)

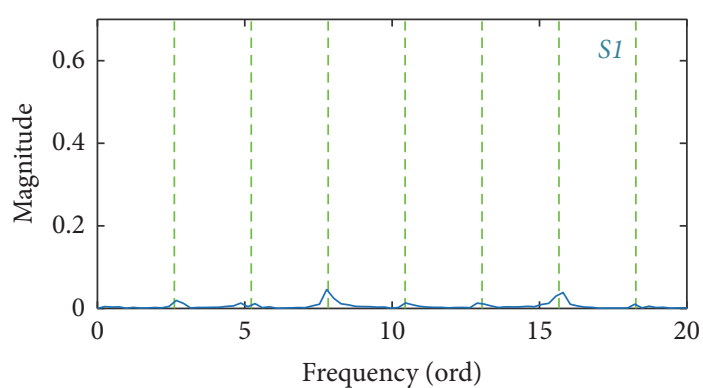

(e)

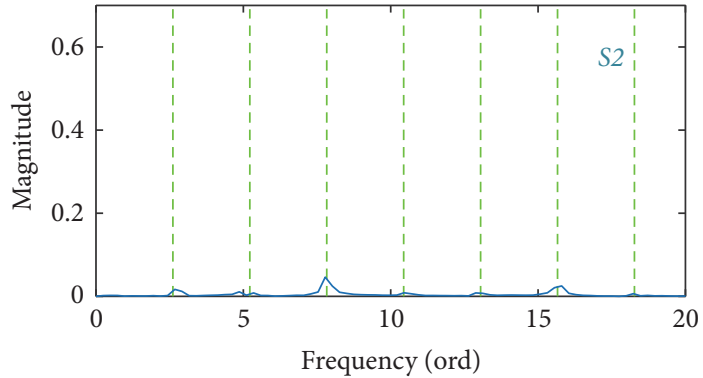

(f)

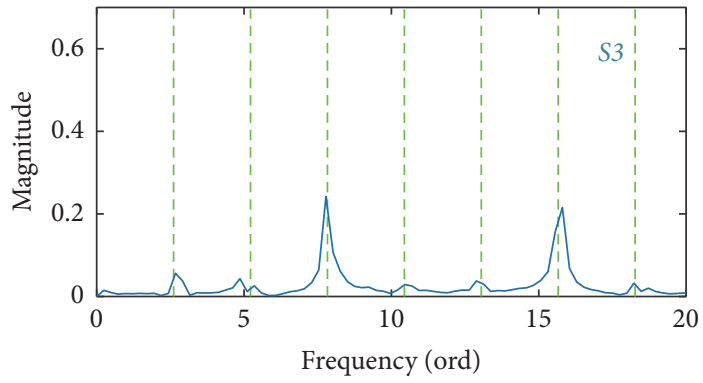

(g)

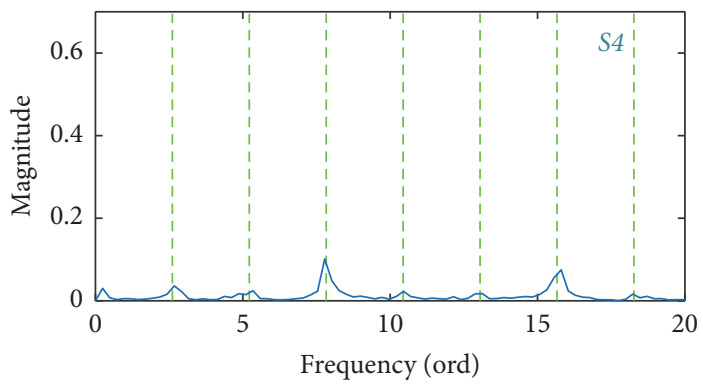

(h)

FIGURE 11: Envelope order spectrums for B1 $(\mathrm{a}-\mathrm{d})$ and for B3 (e-h) for the time domain signals in Figure 10, respectively, from (a) to (d).

With reference to the earlier results in previous section, it is expected that the FRFs obtained from S3 have the highest peak in a frequency range of $4.5 \mathrm{kHz}$ to $6.5 \mathrm{kHz}$, where the fault excites the system resonances. In fact, close inspection of results in Figure 15, in particular comparing envelope spectrums in Figures 8 and 11, reveals that the FRFs, as a transfer path, have a great influence on the amplitudes of generated impact trains, and thus detectability of the corresponding fault frequencies. It is clearly seen that the modulus of the FRF from B1 to S3 in resonance range is approximately ten times greater than other FRFs. This suggests that $\mathrm{S} 3$ can be the ideal location among other sensing locations investigated in this study. According to the FRFs in Figure 15(b), similar increasing trend, but this time with a less difference compared to other FRFs, can also be seen for B3.

In case the experimental FRFs are desired for a similar purpose, with a reasonable assumption, a nearby point can be selected for excitation. In reality, to obtain the experimental FRFs, it is not always possible to excite the system from a point on (or close to) the bearing. 


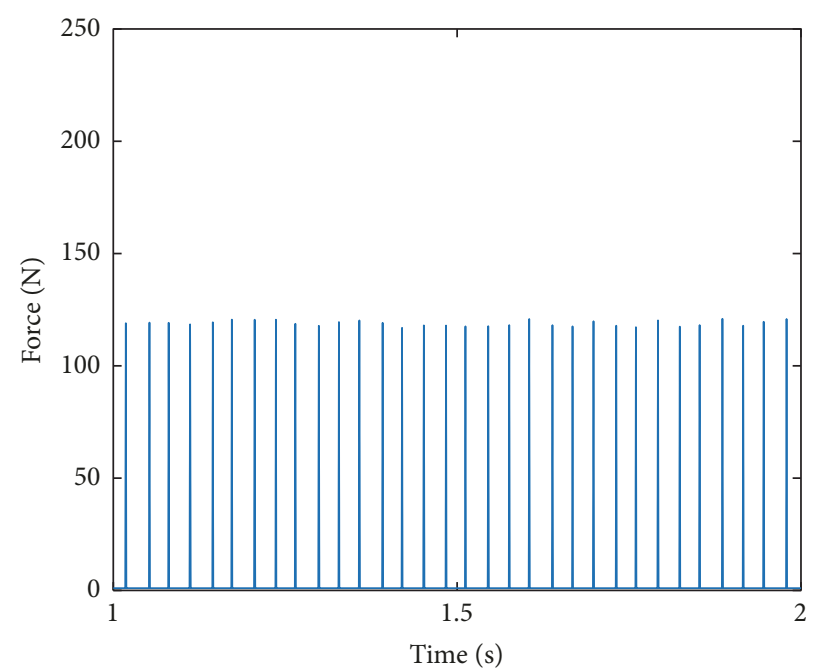

(a)

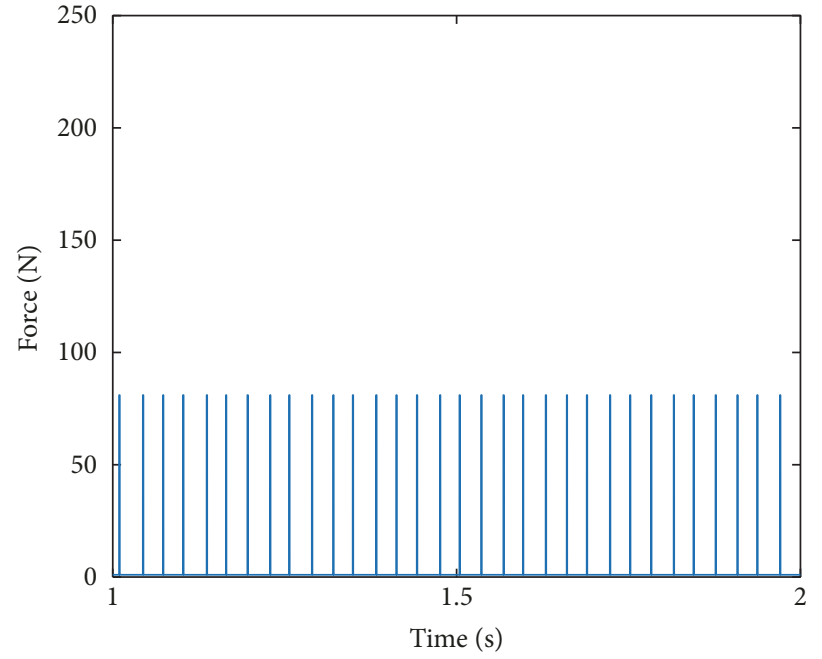

(b)

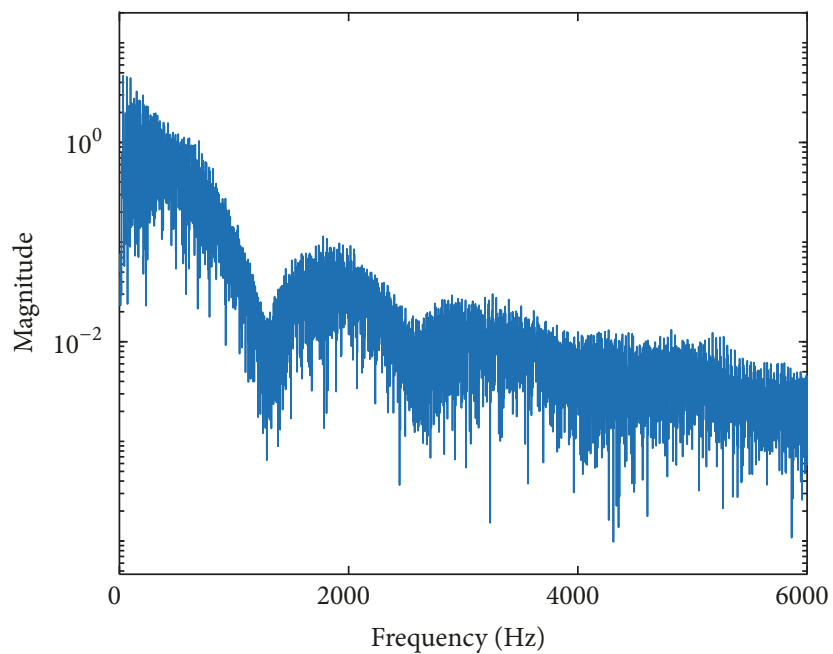

(c)

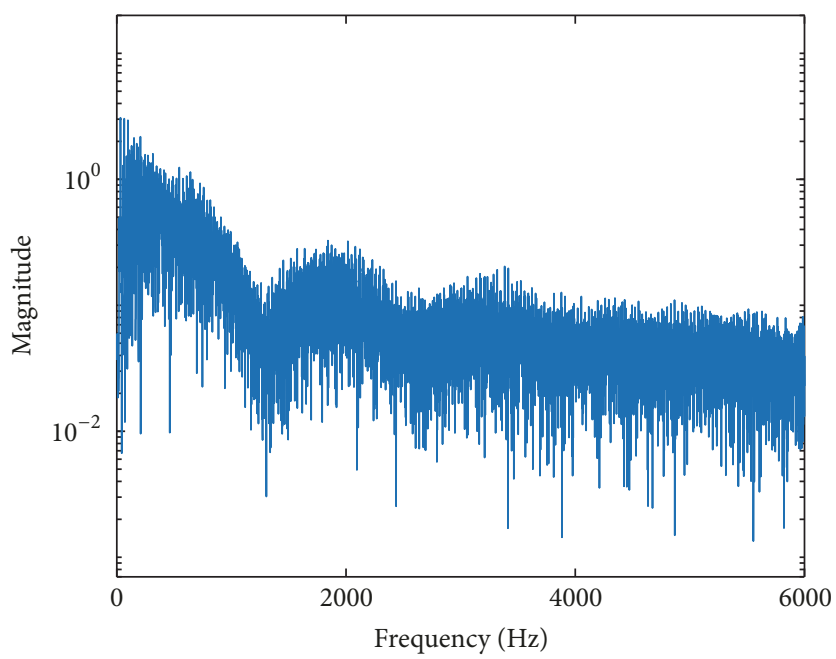

(d)

FIGURE 12: Illustration of the excitation forces to $\mathrm{B} 1$ and $\mathrm{B} 2$ in the time domain in (a) and (b), and in the frequency domain in (c) and (d), respectively.

5.2. Estimation of Bearing Fault Location. As shown in case study 3, the envelope spectrum is unable to detect the fault frequencies of multiple same-type faulty bearings rotating at the same angular velocity. It is shown here that using the structural information obtained from dynamics of the system, it becomes possible to estimate the probable fault location(s).

Figure 16(a) presents an overlaid plot for power spectrums for faulty $\mathrm{B} 1$, from $\mathrm{S} 3$, using $f_{e 1}$ function, each of which with different amount of force magnitude. As seen, all the fault stages in the same bearing excite the same frequency range, but with different magnitudes. In addition, Figure 16(b) presents the power spectrum for case study 3, where both B1 and B2 are faulty. These results in Figure 16 confirm that a localized fault on a specific component in a total system may excite the resonances depending on neighboring components and system dynamics.
Similar to the findings in Figure 15 for B1, while a dominant frequency range (i.e., between $4.5 \mathrm{kHz}$ and $6.5 \mathrm{kHz}$ ) can be seen for different cases having only one faulty bearing (i.e., B1), some other additional dominant frequency ranges appear in the power spectrum for a case having multiple faulty bearings (i.e., B1 and B2). Again, this is an important insight, in particular for the cases where the faulty bearings share same geometry and rotate at the same angular velocity, where envelope spectrum may not be able to diagnose both bearings. To have a better understanding of system resonance areas and the effects of fault excitations due to the localized bearing faults, Figure 17 illustrates the Campbell diagrams, representing both time and frequency domains for case studies 1 and 3. According to the results in Figure 17(a), although the faulty bearing, B1, may excite the total system in a broadband frequency range (with a specific repetition rate), this excitation reaches to its highest amplitude within 


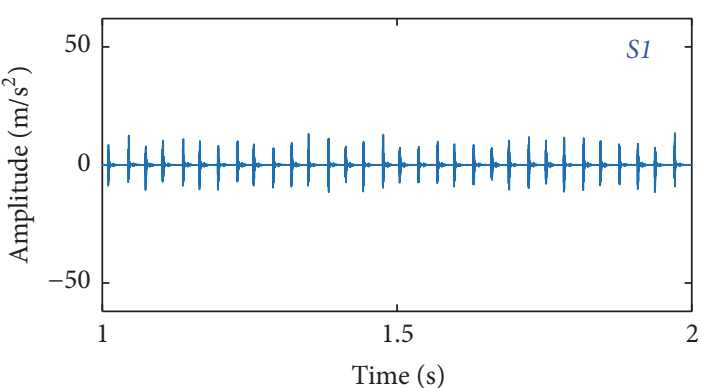

(a)

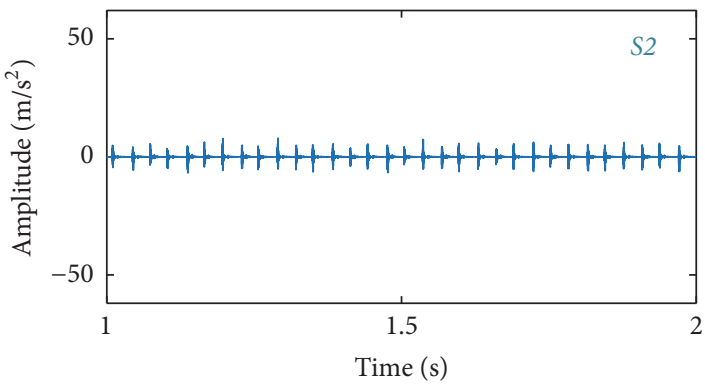

(b)

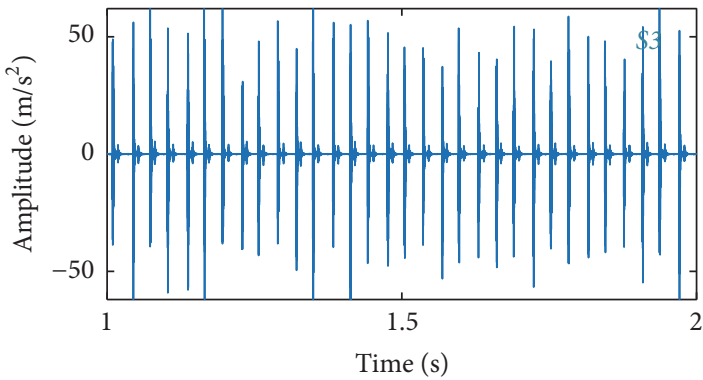

(c)

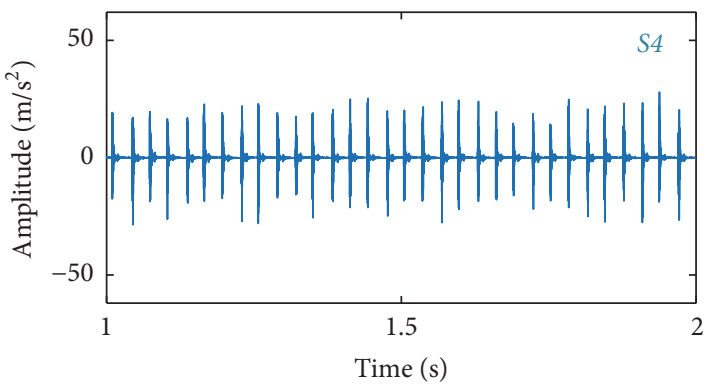

(d)

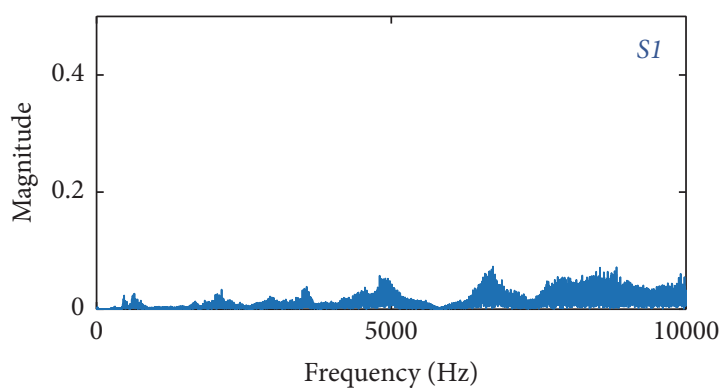

(e)

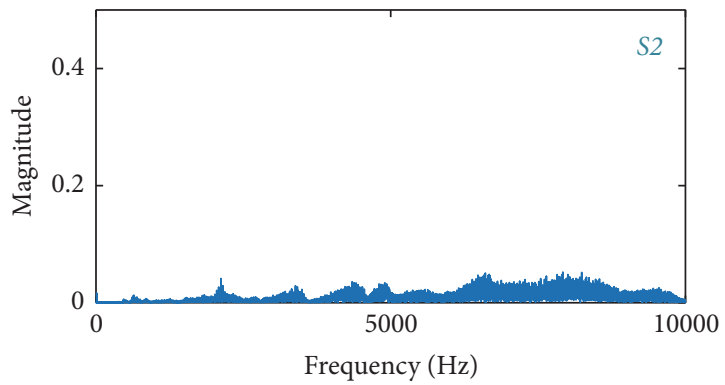

(f)

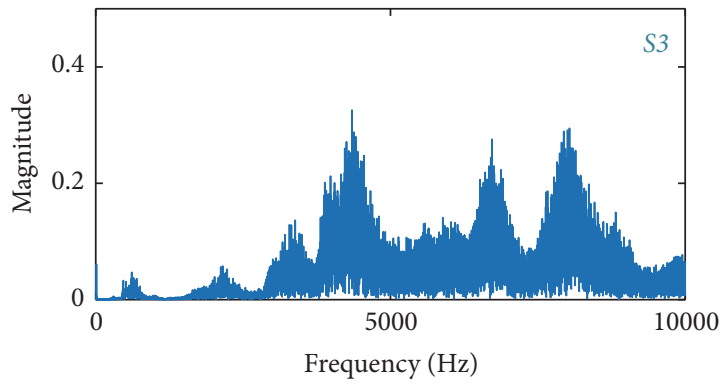

(g)

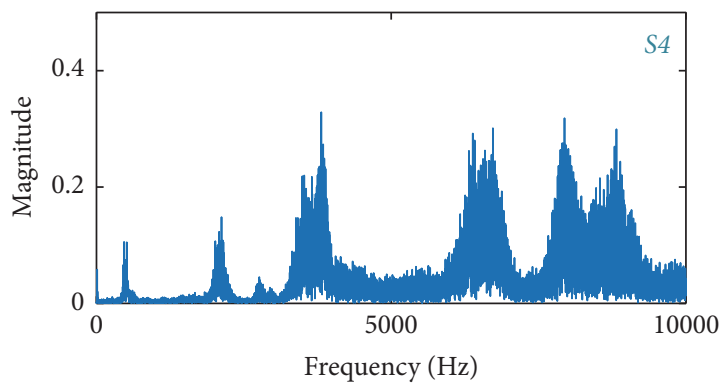

(h)

FIGURE 13: Case study 3: the captured time domain vibration signals from S1 to S4 in (a) to (d), respectively, and their corresponding spectrums in $(\mathrm{e})$ to $(\mathrm{h})$.

a frequency range between $4.5 \mathrm{kHz}$ and $6.5 \mathrm{kHz}$ for case study 1. Similar insights are obtained in Figure 17(b) for case study 3 , but this time two similar faulty bearings rotating at the same speed, B1 and B2, produce highest excitations in distinct frequency ranges. The comparison of results of both case studies here may confirm the existence of another bearing fault with the same repetition frequency, which may lead to a conclusion of existence a fault on B2. Note that, in practice, mechanical faults may lead to excitation of resonances in different frequency bands; however, the numerically generated FRFs excited from a point on the B2, which are shown in Figure 18, suggest that this specific system shows its highest sensitivities to the excitations from B2 in two frequency ranges, one around $4 \mathrm{kHz}$ and one around $8 \mathrm{kHz}$, for S3 and S4. Similar results are previously shown in Figure 14 for case study 3.

Until now, initial fault stages on bearings and their effects on vibration trend of the system are investigated. Here, 


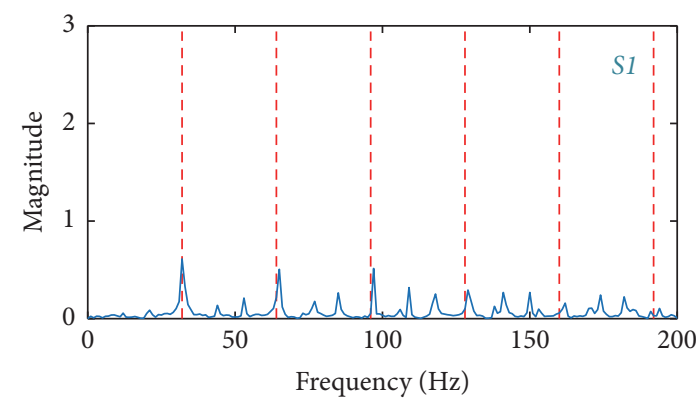

(a)

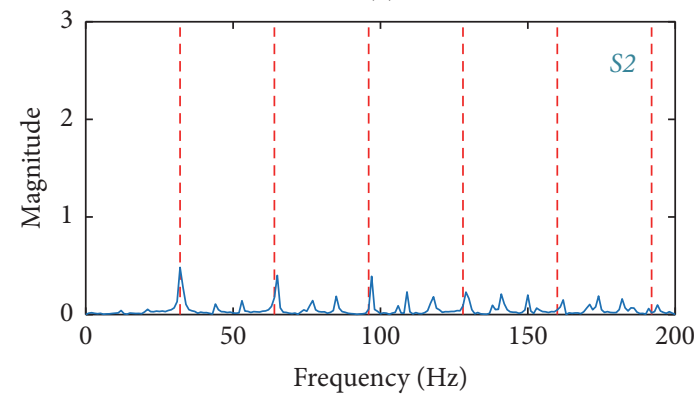

(b)

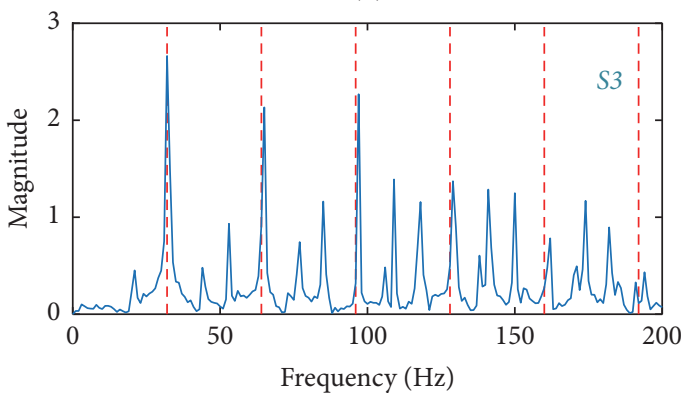

(c)

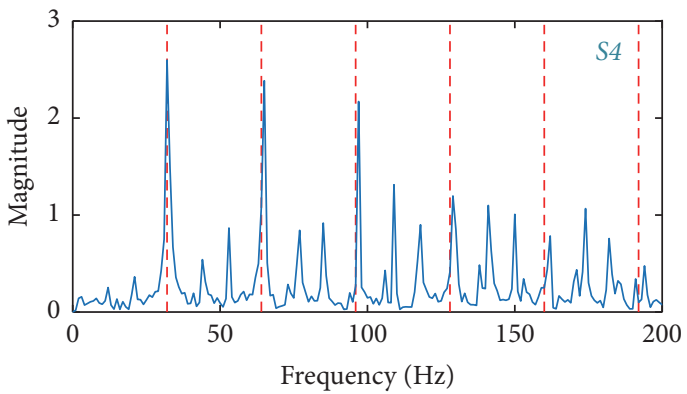

(d)

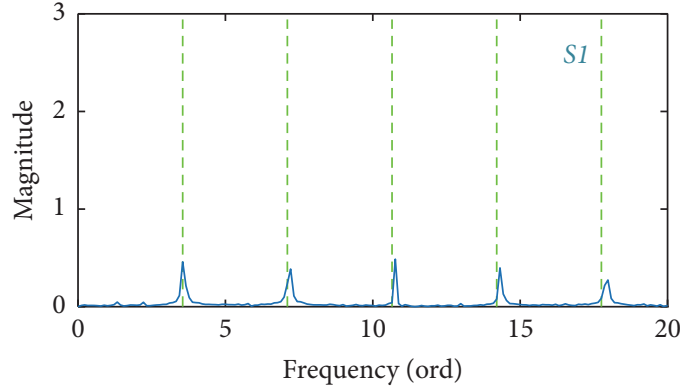

(e)

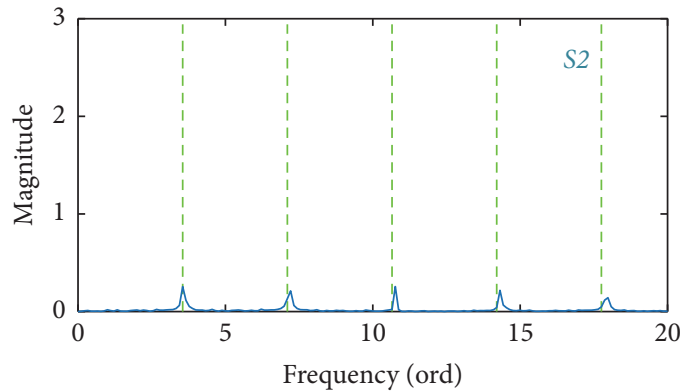

(f)

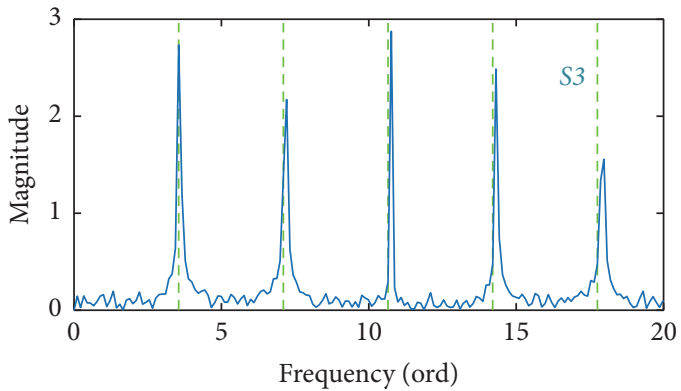

(g)

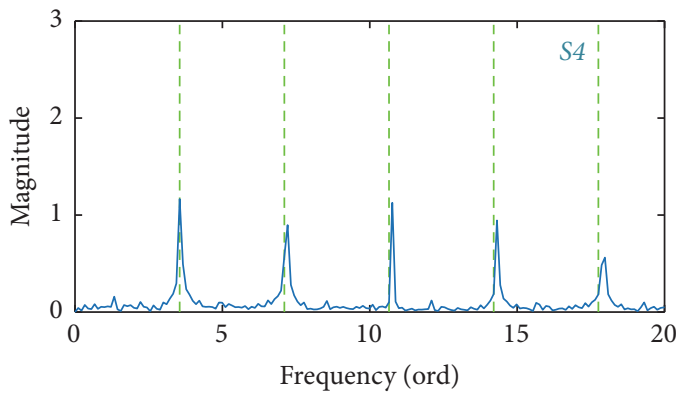

(h)

FIGURE 14: Envelope spectrums (a-d) and envelope order spectrums (e-h) for the time domain signals in Figure 13, respectively, from (a) to (d).

using other two excitation functions, shown in Figure 2, two other fault stages are numerically analyzed in terms of their envelope spectrums. Figure 19 shows an overlaid plot for two envelope spectrums obtained from S3, as a desired sensor position. This is a comparison of a semilocalized fault and a semidistributed fault. As seen, while the fault frequency, BPFO, and its harmonics are visible and sharp for a medium-size fault, they become subtle and difficult to detect for a large-size bearing fault with a more distributed faulty area. This is an expected result, where in reality, when bearing faults grow, they may lose their sharpness by passing the roller elements over time. This example once again illustrates the great importance of detection of bearing faults as early as possible to avoid resulting complications.

\section{Concluding Remarks and Future Works}

6.1. Concluding Remarks. (i) In the present paper, in accordance with the purpose of improving capabilities and 


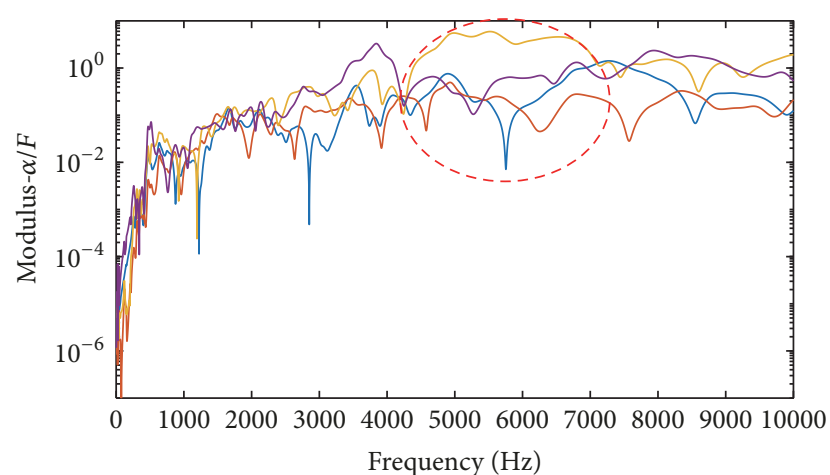

Sensor location

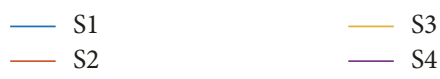

(a)

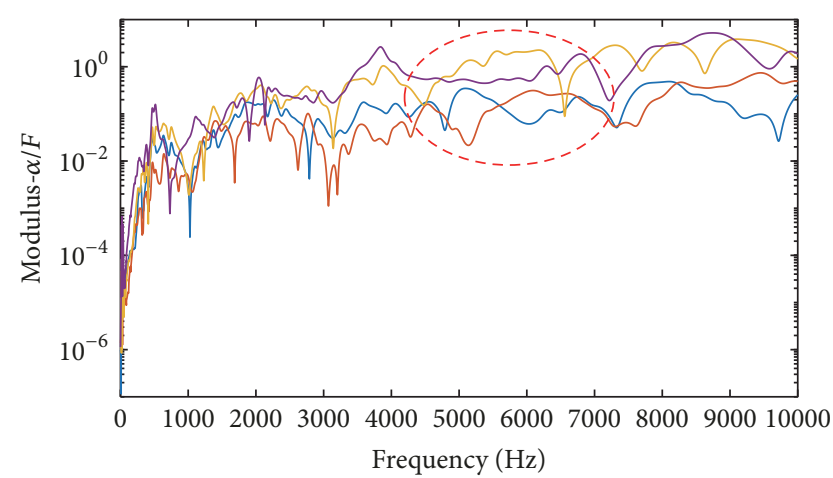

Sensor location

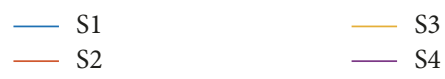

(b)

FIGURE 15: Simulated FRFs obtained in four different sensor locations, exciting from (a) B1 and (b) B3.

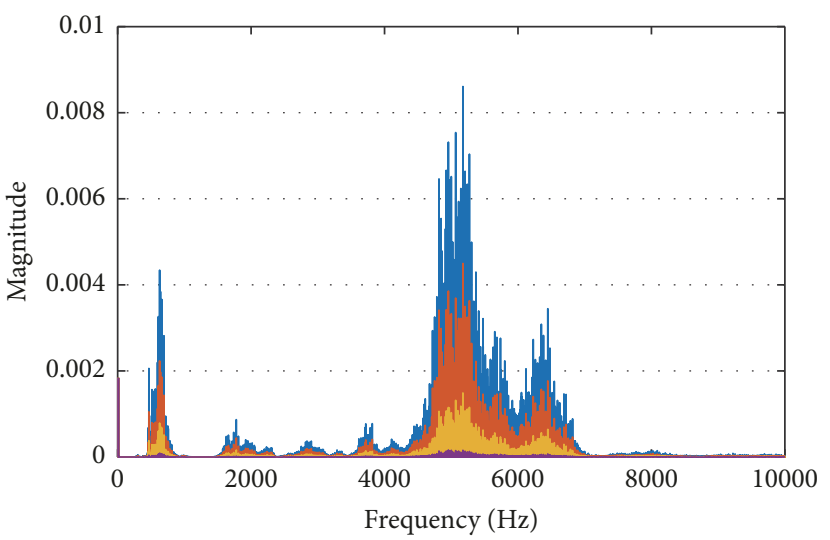

The excitation force function: $f_{e 1}(\theta)$

$280[\mathrm{~N}]$ on $\mathrm{B} 1$

$200[\mathrm{~N}]$ on $\mathrm{B} 1$

(a)

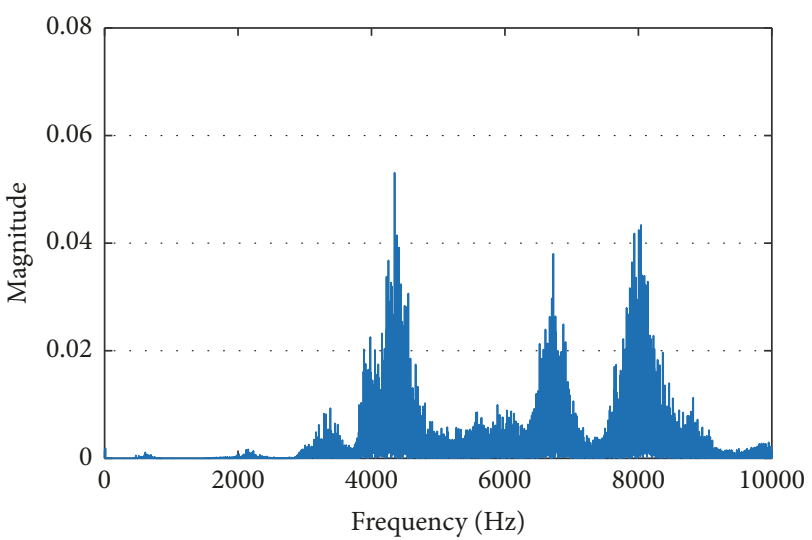

The excitation force function: $f_{e 1}(\theta)$

- $120[\mathrm{~N}]$ on $\mathrm{B} 1 \& 80[\mathrm{~N}]$ on $\mathrm{B} 2$

(b)

FIGURE 16: The power spectrum of the captured signal from S3 for (a) single faulty bearing (b) multiple faulty bearings.

reliabilities of the present vibration-based condition monitoring and fault detection systems for complex mechanical machines/structures in general, and for drivetrains in automotive engineering in particular, a new approach based on combination of numerical modelling and signal processing techniques is developed and investigated in some detail.

(ii) For the objectives of this study, an experimentally validated MBS model of the drivetrain of an electrified vehicle is used as a tool for numerical investigations as well as for illustrating the relations between dynamics of the system and rolling bearing fault signals.

(iii) Three various case studies are defined first and investigated in terms of their vibration responses. It is shown and seen that using only frequency domain information of the measured data may lead to some missed detection, for example, in monitoring of the nearby components with similar geometric details.

(iv) The benefits and limitations of the well-known envelope analysis in automotive engineering applications are highlighted and discussed. A combination of the fields structural dynamics and signal processing, to overcome the available difficulties in finding a desired sensor placement and fault location(s) for the purpose of condition monitoring, is developed. It is shown that using FRFs, as the transfer path functions, delivers a new degree-of-freedom for CM systems. These insights may even be beneficial and useful for further process such as source separation purposes.

(v) Finally, the results from this study show that the approach of analyzing and fault identifying the complex systems using a corresponding MBS model can also be 


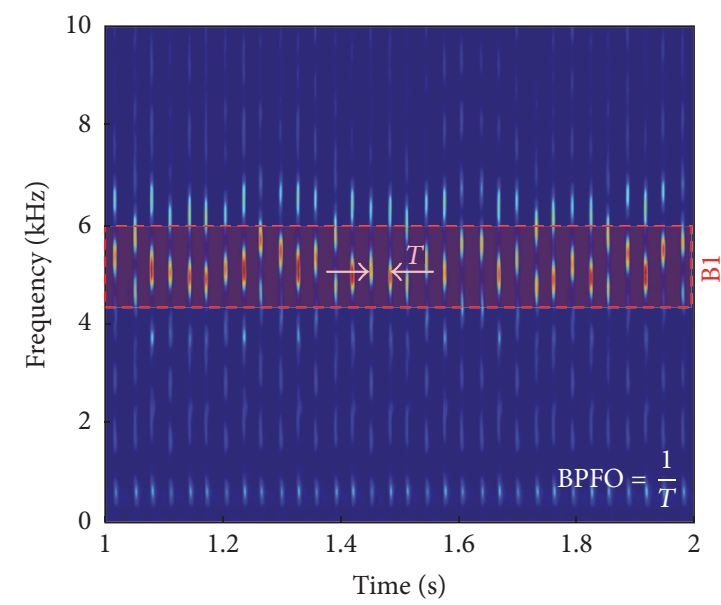

(a)

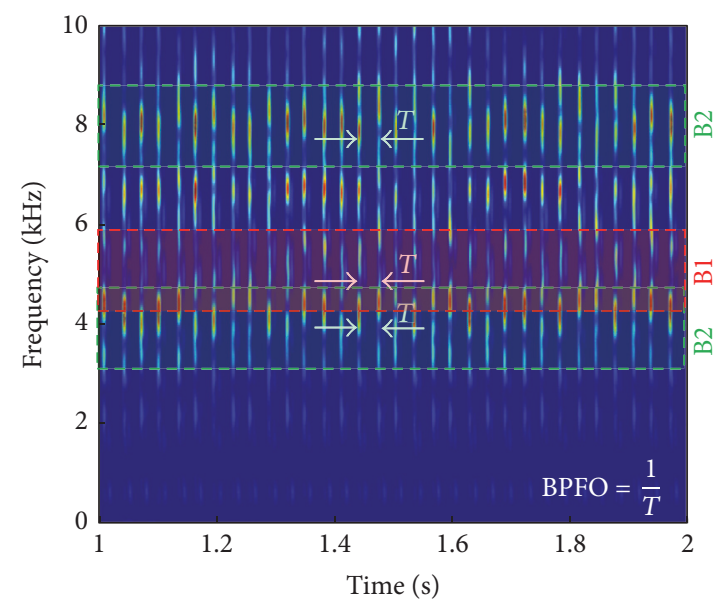

(b)

FIGURE 17: Time-frequency representations of the captured signals from S3 for (a) case study 1: single faulty bearing and (b) case study 3: multiple faulty bearings.

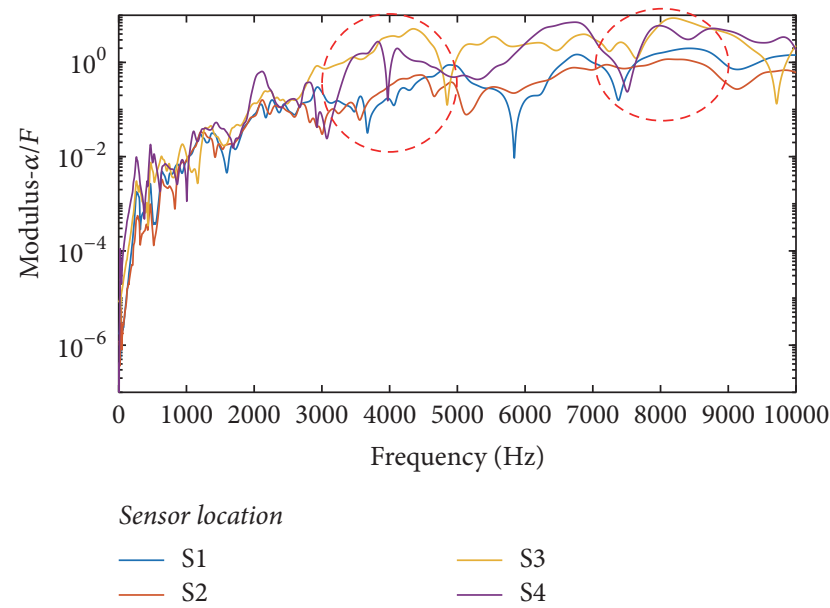

FIGURE 18: Simulated FRFs obtained in four different sensor locations exciting from $\mathrm{B} 2$.

employed as a quality control tool for manufacturers for testing purposes.

6.2. Future Works. In terms of validation and more detailed investigations, the proposed and used methodology needs to be studied and mature further for other complex systems with different faulty cases (e.g., gear stages). In this regard, the present study should be considered as a basis for more comprehensive studies on combination of the fields vibration-based fault diagnosis and numerical modelling. Future works will study a mechanical system having a wide range of mechanical faults.

\section{Conflicts of Interest}

The authors declare that there are no conflicts of interest regarding the publication of this article.

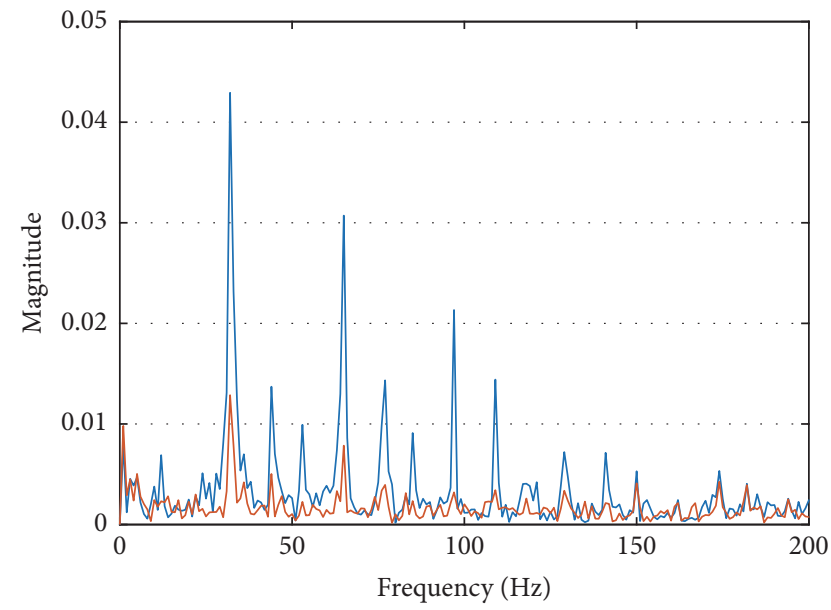

Excitation forces applied to B1

- $100[\mathrm{~N}]$ using $f_{e 2}(\theta)$

$80[\mathrm{~N}]$ using $f_{e 3}(\theta)$

FIGURE 19: Envelope spectrums for two various excitations.

\section{References}

[1] R. Golafshan, M. Wegerhoff, G. Jacobs, and A. I. Ozay, "Vibration-based Condition Monitoring for Wind Turbines: Applications of Singular Value Decomposition," in Proceedings of the 17th Drive Train Technology Conference (ATK2017), Aachen, Germany, march 2017.

[2] R. B. Randall and J. Antoni, "Rolling element bearing diagnostics-a tutorial," Mechanical Systems and Signal Processing, vol. 25, no. 2, pp. 485-520, 2011.

[3] D. De Klerk and D. J. Rixen, "Component transfer path analysis method with compensation for test bench dynamics," Mechanical Systems \& Signal Processing, vol. 24, no. 6, pp. 1693-1710, 2010.

[4] J. Plunt, "Examples of using transfer path analysis (TPA) together with CAE-models to diagnose and find solutions for 
NVH problems late in the vehicle development process," SAE Technical Papers, 2005.

[5] Z. Li, M. Q. Feng, L. Luo, D. Feng, and X. Xu, "Statistical analysis of modal parameters of a suspension bridge based on Bayesian spectral density approach and SHM data," Mechanical Systems and Signal Processing, vol. 98, pp. 352-367, 2018.

[6] R. Klein, "Comparison of methods for separating vibration sources in rotating machinery," Mechanical Systems and Signal Processing, vol. 97, pp. 20-32, 2017.

[7] R. B. Randall and W. Smith, "New cepstral methods for the diagnosis of gear and bearing faults under variable speed conditions," in Proceedings of the 23rd International Congress on Sound and Vibration (ICSV23), Athens, Greece, July 2016.

[8] M. M. Rezaei, M. Behzad, H. Moradi, and H. Haddadpour, "Modal-based damage identification for the nonlinear model of modern wind turbine blade," Journal of Renewable Energy, vol. 94, pp. 391-409, 2016.

[9] A. Bajric, J. Høgsberg, and F. Rüdinger, "Evaluation of damping estimates by automated Operational Modal Analysis for offshore wind turbine tower vibrations," Renewable Energy, vol. 116, pp. 153-163, 2017.

[10] P. Drichel, M. Wegerhoff, R. Schelenz, and G. Jacobs, "Modeling and electric vehicle powertrain and analysis of vibration characteristics," in Proceedings of the Torsional Vibaration Symposium, Salzburg, Austria, 2014.

[11] J. Berroth, G. Jacobs, T. Kroll, and R. Schelenz, "Investigation on pitch system loads by means of an integral multi body simulation approach," Journal of Physics: Conference Series, vol. 75, Article ID 112002, 2016.

[12] A. Rasekhi Nejad, P. F. Odgaard, Z. Gao, and T. Moan, "A prognostic method for fault detection in wind turbine drivetrains," Engineering Failure Analysis, vol. 42, pp. 324-336, 2014.

[13] D. Gerhard, "Product Lifecycle Management Challenges of CPPS," in Multi-Disciplinary Engineering for Cyber-Physical Production Systems, pp. 89-110, Springer International Publishing AG, Cham, Switzerland, 2017.

[14] S. Boschert and R. Rosen, "Digital Twin-The Simulation Aspect," in Mechatronic Futures, pp. 59-74, Springer International Publishing AG, Cham, Switzerland, 2016.

[15] N. Tandon and A. Choudhury, "Review of vibration and acoustic measurement methods for the detection of defects in rolling element bearings," Tribology International, vol. 32, no. 8, pp. 469-480, 1999.

[16] M. Cerrada, R.-V. Sánchez, C. Li et al., "A review on data-driven fault severity assessment in rolling bearings," Mechanical Systems and Signal Processing, vol. 99, pp. 169-196, 2018.

[17] P. D. McFadden and J. D. Smith, "Model for the vibration produced by a single point defect in a rolling element bearing," Journal of Sound and Vibration, vol. 96, no. 1, pp. 69-82, 1984.

[18] W. Cheng, Z. Zhang, S. Lee, and Z. He, "Investigations of denoising source separation technique and its application to source separation and identification of mechanical vibration signals," Journal of Vibration and Control, vol. 20, no. 14, pp. 2100-2117, 2013.

[19] R. Golafshan and K. Yuce Sanliturk, "SVD and Hankel matrix based de-noising approach for ball bearing fault detection and its assessment using artificial faults," Mechanical Systems and Signal Processing, vol. 70-71, pp. 36-50, 2016.

[20] J. Antoni, “The spectral kurtosis: a useful tool for characterising non-stationary signals," Mechanical Systems and Signal Processing, vol. 20, no. 2, pp. 282-307, 2006.
[21] M. Giebeler-Müller, P. Drichel, M. Jaeger et al., "Comprehensive model for the assessment of the NVH-behavior of electric vehicles," in Proceedings of the Aachen Acoustics Colloquium (AAC 2017), Aachen, Germany, 2017.

[22] J. Slavic, A. Brkovic, and M. Boltezar, "Typical bearing-fault rating using force measurements: application to real data," Journal of Vibration and Control, vol. 17, no. 14, pp. 2164-2174, 2011.

[23] Z. Kiral and H. Karagulle, "Simulation and analysis of vibration signals generated by rolling element bearing with defects," Tribology International, vol. 36, no. 9, pp. 667-678, 2003. 


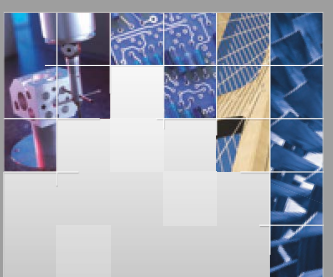

\section{Enfincering}
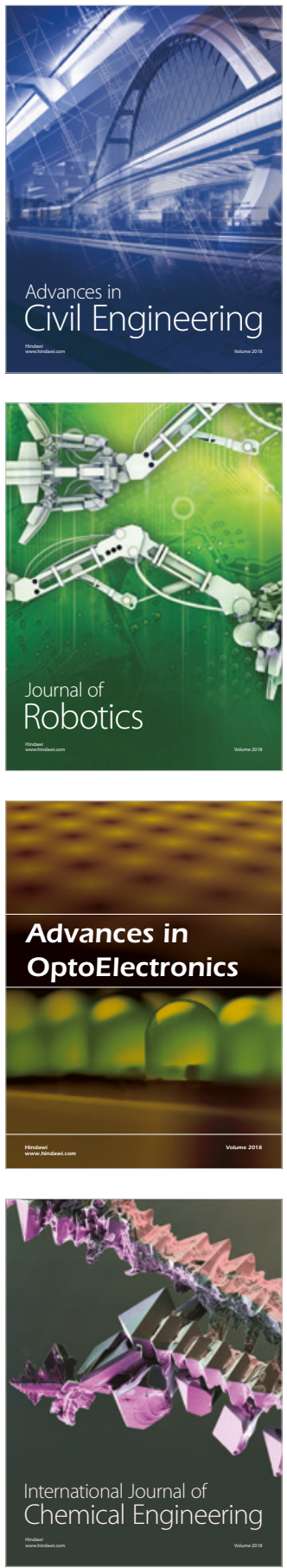

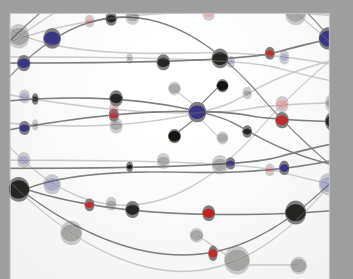

\section{Rotating \\ Machinery}

The Scientific World Journal

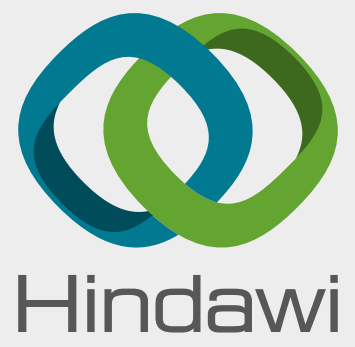

Submit your manuscripts at

www.hindawi.com
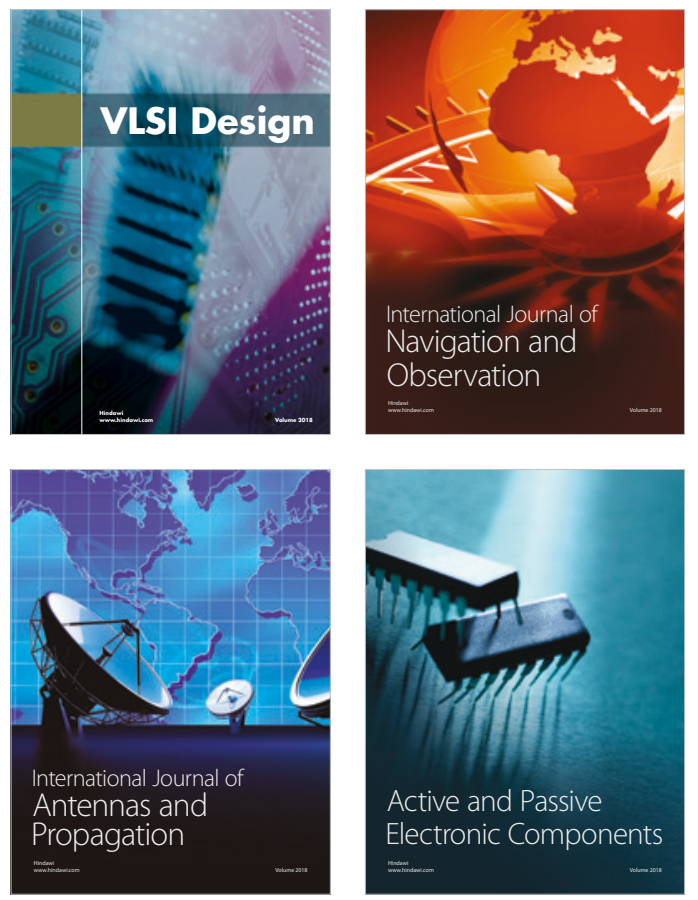
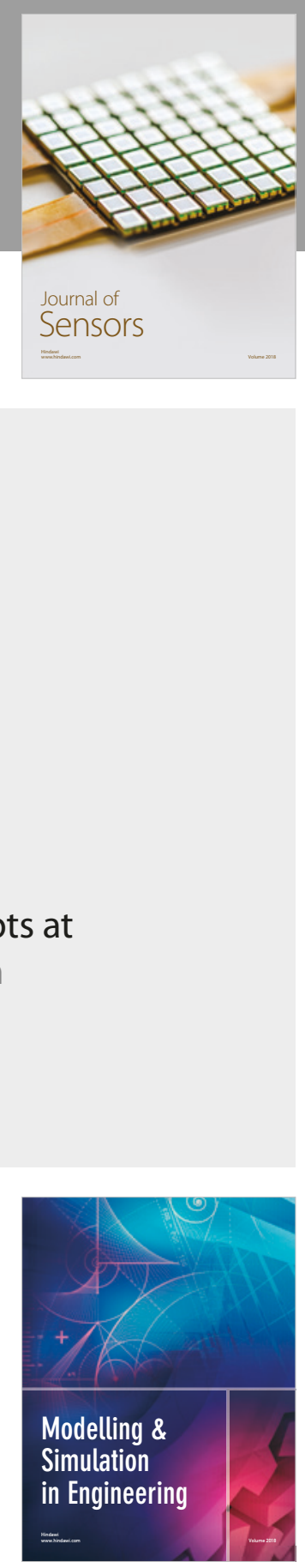

\section{Advances \\ Multimedia}
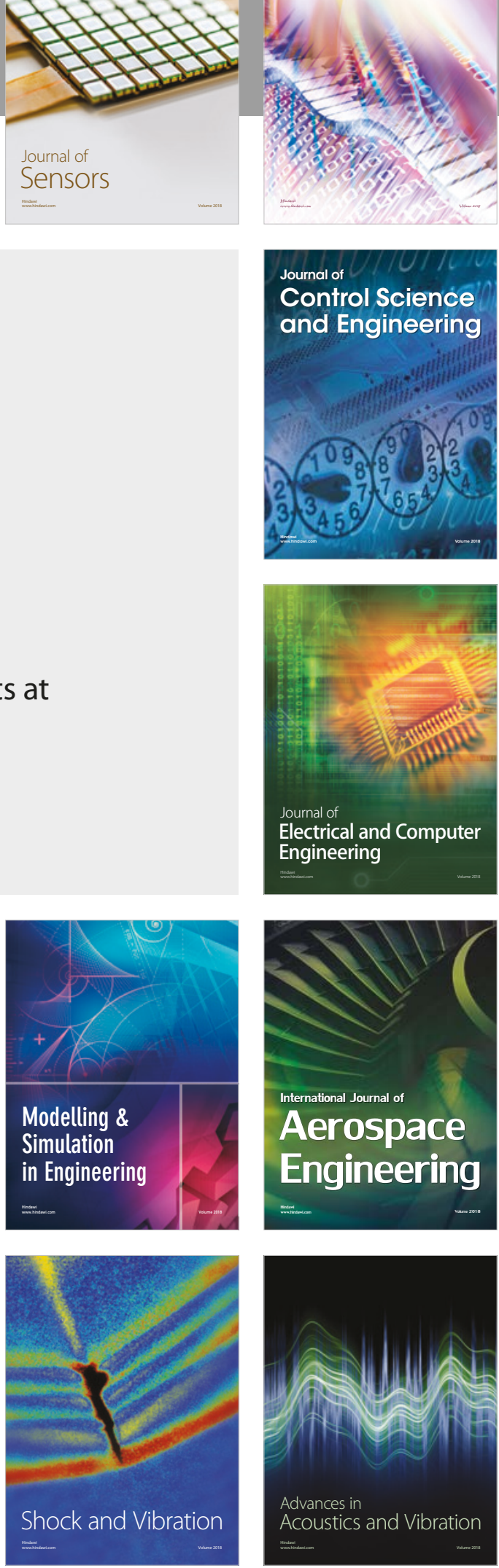